\title{
THE BOUNDARY OF THE MILNOR FIBRE OF CERTAIN NON-ISOLATED SINGULARITIES
}

\author{
ANDRÁS NÉMETHI AND GERGŐ PINTÉR
}

\begin{abstract}
Let $\Phi:\left(\mathbb{C}^{2}, 0\right) \rightarrow\left(\mathbb{C}^{3}, 0\right)$ be a finitely determined complex analytic germ and let $(\{f=0\}, 0)$ be the reduced equation of its image, a non-isolated hypersurface singularity. We provide the plumbing graph of the boundary of the Milnor fiber of $f$ from the double-point-geometry of $\Phi$.
\end{abstract}

\section{INTRODUCTION}

The Milnor fiber of an isolated hypersurface surface singularity is well studied and it is rather well understood. It has the homotopy type of a bouquet of 2 -spheres, it is an oriented smooth 4-manifold whose boundary is diffeomorphic with the link of the singular germ and also with the boundary of (any) resolution of the germ [13]. This boundary is a plumbed 3-manifold and one can take as a plumbing graph any of the resolution graphs. It is the basic bridge between the Milnor fiber and the resolution (both of them being complex analytic fillings of it), and this way produces several nice formulas connecting the invariants of these fillings. Here primarily we think about formulas of Laufer [5] or Durfee [3] and their generalizations, see e.g. [27].

For non-isolated hypersurface singularities in $\left(\mathbb{C}^{3}, 0\right)$ the situation is more complicated. First of all, the link of the germ is not smooth, hence the boundary of the Milnor fiber cannot be isomorphic with it. Moreover, a (any) resolution is in fact the resolution of the normalization (which might contain considerable less information than what one needs in order to recover the Milnor fiber $F$, or the Milnor fiber boundary $\partial F$ ), see e.g. [25, 22]. For example (see the present article), it can happen that the normalization is smooth, while $\partial F$ is rather complicated. However, the boundary of the Milnor fiber is still a plumbed 3manifold, and one expects that its plumbing graph codifies considerable information about the germ. $\partial F$ can be obtained by surgery of two pieces: one of them is the boundary of the resolution of the normalization, the other one is related with the transversal singularities associated with the singular curves of the hypersurface singularity $[25,22,11]$. In particular, the boundary of the Milnor fiber plays the same crucial role as in the isolated singularity case (in fact, it is the unique object in this case, which might fulfill this role): it is the first step in the description of the Milnor fiber, and it is the bridge in the direction of the resolution and the transversal types of the components of the singular locus.

[22] presents a general algorithm, which provides the boundary of the Milnor fiber $\partial F$ for any non-isolated hypersurface singularity in $\left(f^{-1}(0), 0\right) \subset\left(\mathbb{C}^{3}, 0\right)$. However, this algorithm uses (some information from) the embedded resolution of this pair, hence it is rather technical and in concrete examples is rather computational. Therefore, for particular families

2010 Mathematics Subject Classification. Primary. 32S05, 32S25, 32S50, 57M27, Secondary. 14Bxx, 32Sxx, 57R57, 55N35.

Key words and phrases. hypersurface singularities, non-isolated singularities, links of singularities, Milnor fiber, Seifert 3-manifolds, plumbing graphs, boundary of the Milnor fiber.

Both authors were partially supported by NKFIH Grant 112735 and ERC Adv. Grant LDTBud of A. Stipsicz at Rényi Institute of Math., Budapest. GP was also supported by 'Lendület' program 'LTDBud' at Rényi Institute. 
of singularities it is preferable to find more direct description of the plumbing graph of $\partial F$ directly from the peculiar intrinsic geometry of the germ. For several examples in the literature see e.g. [22] (homogeneous singularities, cylinders of plane curves, $f=z f^{\prime}(x, y)$, $\left.f=f^{\prime}\left(x^{a} y^{b}, z\right)\right),[26](f=g(x, y)+z h(x, y))$; or for other classes consult also [12] and [1].

In this note we assume that $\left(f^{-1}(0), 0\right)$ is the image of a finitely determined complex analytic germ $\Phi:\left(\mathbb{C}^{2}, 0\right) \rightarrow\left(\mathbb{C}^{3}, 0\right)$. This means that $\Phi$ is a generic immersion off the origin, cf. $[28,16]$. The main result of the article provides the plumbing graph of $\partial F$, as a surgery, starting from the embedded resolution graph of the double points $(D, 0) \subset\left(\mathbb{C}^{2}, 0\right)$. The needed additional pieces, which will be glued to this primary object correspond to certain fiber bundles over $S^{1}$ with fibers the local Milnor fiber of the transversal singularity type (and monodromy the corresponding vertical monodromy). The surgery itself is characterized by some homologically determined integers combined with the newly defined 'vertical index' associated with the irreducible components of the singular locus of $f^{-1}(0)$.

We believe that the present method can serve as a prototype for further more general families as well.

The structure of the article is the following. In Section 2 we introduce the notations, in Section 3 we describe by several characterizations the surgery pieces associated with the components of the singular locus. In Section 4 we describe the gluing, while the last section contains several concrete examples (the families are taken from the Mond's list of simple germs $[16])$.

Below $\mathbb{Z}_{n}\langle g\rangle$ denotes the cyclic group of order $n$ generated by $g$. We write $\partial_{x_{i}} f$ instead of $\partial f / \partial x_{i}$.

\section{Preliminaries}

2.1. Let $\Phi:\left(\mathbb{C}^{2}, 0\right) \rightarrow\left(\mathbb{C}^{3}, 0\right)$ be a complex analytic germ singular only at the origin. We assume that $\Phi$ is finitely determined. This happens exactly when $\Phi$ is a generic immersion off the origin, that is, off the origin it has only single and double values and at each double value the intersection of the two smooth branches is transversal, cf. [28, Theorem 2.1] or [16, Corollary 1.5].

Write $(X, 0):=(\operatorname{im}(\Phi), 0)$ and let $f:\left(\mathbb{C}^{3}, 0\right) \rightarrow(\mathbb{C}, 0)$ be the reduced equation of $(X, 0)$. Note that $(X, 0)$ is a non-isolated hypersurface singularity, except when $\Phi$ is a regular map (see [21]). We denote by $(\Sigma, 0)=\left(\partial_{x_{1}} f, \partial_{x_{2}} f, \partial_{x_{3}} f\right)^{-1}(0) \subset\left(\mathbb{C}^{3}, 0\right)$ the reduced singular locus of $(X, 0)$ (which equals the closure of the set of double values of $\Phi)$, and by $(D, 0)$ the reduced double point curve $\Phi^{-1}(\Sigma) \subset\left(\mathbb{C}^{2}, 0\right)$. (In fact, the finite determinacy of the germ $\Phi$ is equivalent with the fact that the double point curve is reduced; see e.g. [10].)

Let $B_{\epsilon}^{6}$ be the $\epsilon$-ball in $\mathbb{C}^{3}$ centered at the origin, and $S_{\epsilon}^{5}$ its boundary $\left(\epsilon \in \mathbb{R}_{>0}\right)$. Then for $\epsilon$ sufficiently small $\left(B_{\epsilon}^{6}, 0\right)$ is a Milnor ball for the pair $(\Sigma, 0) \subset(X, 0)$, and, furthermore, $\mathfrak{B}_{\epsilon}:=\Phi^{-1}\left(B_{\epsilon}^{6}\right)$ is a (non-metric) $C^{\infty}$ ball in $\left(\mathbb{C}^{2}, 0\right)$, which might serve as a Milnor ball for $(D, 0)$, cf. [7]. We set $\mathfrak{S}^{3}=\Phi^{-1}\left(S_{\epsilon}^{5}\right)=\partial \mathfrak{B}_{\epsilon}$, diffeomorphic to $S^{3}$, and we treat it as the usual Milnor-ball boundary 3 -sphere. Recall that the immersion associated with $\Phi$ at the level of local neighbourhood boundaries is $\left.\Phi\right|_{\mathfrak{S}^{3}}: \mathfrak{S}^{3} \rightarrow S^{5}[21]$.

2.2. Components and links of $\Sigma$ and $D$. Let $\Upsilon \subset S_{\epsilon}^{5}$ be the link of $\Sigma$. It is exactly the set of double values of $\left.\Phi\right|_{\mathfrak{S}^{3}}$. Let $L=\Phi^{-1}(\Upsilon) \subset \mathfrak{S}^{3}$ denote the set of double points of $\left.\Phi\right|_{\mathfrak{S}^{3}}$, that is, $L \subset \mathfrak{S}^{3}$ is the link of $D$. Assume that the reduced equation of $D$ is $d:\left(\mathbb{C}^{2}, 0\right) \rightarrow(\mathbb{C}, 0)$, whose irreducible decomposition is $d=\Pi_{i=1}^{l} d_{i}$. The irreducible components of $D$ are denoted by $\left(D_{i}, 0\right)=d_{i}^{-1}(0)$ and their link components in $L$ by $L_{i}$, $1 \leq i \leq l$. $D$ is equipped with an involution $\iota: D \rightarrow D$ which pairs the double points. $\left.\iota\right|_{L}$ induces a permutation (pairing) $\sigma$ of $\{1,2, \ldots, l\}$, such that $\iota\left(L_{i}\right)=L_{\sigma(i)}$. Moreover, $\left.\Phi\right|_{L}: L \rightarrow \Upsilon$ is a double covering with $\Phi\left(L_{i}\right)=\Phi\left(L_{\sigma(i)}\right)$. If $i=\sigma(i)$ for some $i$, then $\left.\Phi\right|_{L_{i}}$ is 
a nontrivial double covering of its image, while above the other components the covering is trivial. Let $J$ be the set of pairs $\{i, \sigma(i)\}(1 \leq i \leq l)$, it is the index set of the components of $\Upsilon$; they will be denoted by $\left\{\Upsilon_{j}\right\}_{j \in J}$.

All link components are considered with their natural orientations.

2.3. The embedded resolution of $(D, 0) \subset\left(\mathbb{C}^{2}, 0\right)$. Let $\pi:\left(\widetilde{\mathbb{C}^{2}}, E\right) \rightarrow\left(\mathbb{C}^{2}, 0\right)$ be a good embedded resolution of $(D, 0), E=\pi^{-1}(0)$ the reduced exceptional curve, $\bigcup_{v \in V} E_{v}$ the irreducible components of $E$, each diffeomorphic to $\mathbb{C P}^{1}$ intersecting each other transversally. Let $e_{v} \in \mathbb{Z}$ denote the self-intersection number of $E_{v}$ in $\widetilde{\mathbb{C}^{2}}$. Clearly $e_{v}<0$ for all $v$.

We denote the strict transform $\overline{(d \circ \pi)^{-1}(0) \backslash E} \subset \widetilde{\mathbb{C}^{2}}$ of $D$ by $\tilde{D}$, and its components by $\tilde{D}_{i}, 1 \leq i \leq l$. Each $\tilde{D}_{i}$ intersects (transversally) only one exceptional component, say $E_{v(i)}$.

The total transform of $D_{i}$ is the divisor $\operatorname{div}\left(d_{i} \circ \pi\right)=\Sigma_{v \in V} m_{i}(v) \cdot E_{v}+\tilde{D}_{i}$, where the multiplicity $m_{i}(v) \in \mathbb{Z}_{>0}$ is the vanishing order of $d_{i} \circ \pi$ along $E_{v}$.

Let $\Gamma$ be the embedded resolution graph of $(D, 0) \subset\left(\mathbb{C}^{2}, 0\right)$ associated with the resolution $\pi$. The strict transforms $\left\{\tilde{D}_{i}\right\}_{i=1}^{l}$ will be codified (as usual) by arrowhead vertexes $\left\{a_{i}\right\}_{i=1}^{l}$.

The multiplicities $m_{i}(v)(v \in V, 1 \leq i \leq l)$ are determined by $\Gamma$ via the identities (see e.g. $[4,20])$

$$
\Sigma_{v \in V} m_{i}(v)\left(E_{v} \cdot E_{w}\right)+\left(\tilde{D}_{i} \cdot E_{w}\right)=0 \text { for all } w \in V
$$

\section{THE MANIFOLD $Y$}

In the construction of the boundary of the Milnor fiber we will need a special 3-manifold with torus boundary. Its several realizations and properties will be discussed in this section.

In the sequel $S^{1}$ (as the boundary of the unit disc of $\mathbb{C}$ ) and the real interval $I:=[-1,1]$ are considered with their natural orientations; ${ }^{-}$denotes the complex conjugation of $\mathbb{C}$.

3.1. The definition of $Y$. Consider the $\mathbb{Z}_{2}$-action on $S^{1} \times S^{1} \times I$ defined by the involution $(x, y, z) \mapsto(-x, \bar{y},-z)$, and define $Y$ as the factor

$$
Y=\frac{S^{1} \times S^{1} \times I}{(x, y, z) \sim(-x, \bar{y},-z)} .
$$

$Y$ is a $3-$ manifold with a boundary diffeomorphic with $S^{1} \times S^{1}$. The projections to different components provide different 'realizations' of $Y$.

(1) The projection to the first coordinate $x$ gives a fibration

$$
\begin{array}{cc}
S^{1} \times I \rightarrow & Y \\
& \downarrow \\
& S^{1}
\end{array}
$$

where the base space $S^{1}=S^{1} /\{x \sim-x\}$ is parametrized by $x^{2}$, and the monodromy diffeomorphism $S^{1} \times I \rightarrow S^{1} \times I$ over the base space is $(y, z) \mapsto(\bar{y},-z)$.

(2) The projection to the first two coordinates $(x, y)$ realizes $Y$ as the total space of a fibration

$$
\begin{aligned}
I \rightarrow & Y \\
& \downarrow \\
& \mathcal{K}
\end{aligned}
$$

with fiber $I$ and base space $\mathcal{K}:=\left(S^{1} \times S^{1}\right) /\{(x, y) \sim(-x, \bar{y})\}$, the Klein bottle. The factorization $S^{1} \times S^{1} \rightarrow \mathcal{K}$ is the orientation double cover of $\mathcal{K}$. In particular, the fibration (3.1.3) is the segment bundle of the orientation line bundle of $\mathcal{K}$, hence the orientation 
double cover of $\mathcal{K}$ is realized also by the restriction of the bundle map to the boundary $\partial Y \approx S^{1} \times S^{1}$.

(3) The projection to the $(x, z)$ coordinates realizes $Y$ as the total space of a fibration

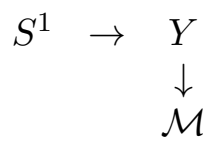

over the base space $\mathcal{M}:=\left(S^{1} \times I\right) /\{(x, z) \sim(-x,-z)\}$, the Möbius band. In this way, $Y$ appears as the tangent circle bundle of $\mathcal{M}$, i.e. as the sub-bundle of the tangent bundle $T \mathcal{M}$ consisting of unit tangent vectors. This follows from the fact that both circle bundles have the same monodromy map along the midline of $\mathcal{M}$, namely $S^{1} \rightarrow S^{1}, y \mapsto \bar{y}$.

(4) The projection to the $(y, z)$ coordinates realizes $Y$ as the total space of a projection

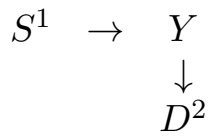

to the base space $\left(S^{1} \times I\right) /\{(y, z) \sim(\bar{y},-z)\}$, the 2-disc $D^{2}$. Although the involution $(y, z) \mapsto(\bar{y},-z)$ has two fix points $(-1,0)$ and $(1,0)$, the factor $D^{2}$ can be smoothed. However, the projection $Y \rightarrow D^{2}$ is not a locally trivial fibration: it is a Seifert fibration with two exceptional fibers sitting above $(-1,0)$ and $(1,0)$. We will refer to this $S^{1}$-fibration as the canonical Seifert fibration of $Y$.

The Seifert invariants of the exceptional fibers can be calculated as in p. 307 of [23]. The two exceptional fibers of the canonical Seifert fibration (3.1.5) can be seen in the projection (3) as well: they correspond to the tangent vectors of the midline. On the other hand, a generic orbit consists of those unit tangent vectors of $\mathcal{M}$, which form non-zero angle $\pm \alpha$ (with $\alpha$ fixed) with the midline. Thus, a generic fiber in a neighbourhood of an exceptional fiber goes around twice and both Seifert invariants are $(2,1)$. Hence, cf. [23], a plumbing graph of $Y$ is:

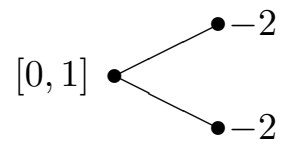

Here $[0,1]$ denotes a genus 0 core-space with one disc removed. The Euler number of the $S^{1}$-bundle corresponding to the middle vertex is irrelevant, the resulted 3-manifolds with boundary are diffeomorphic with each other (hence with $Y$ too). However, the restriction of the canonical Seifert fibration to $\partial Y \simeq S^{1} \times S^{1}$ determines an $S^{1}$-fibration of the boundary. Moreover, $Y$ admits a unique closed Seifert 3-manifold $\bar{Y}$, from which $Y$ can be obtained by omitting a tubular neighborhood of a generic fibre (that is, $\bar{Y}$ is obtained by extending the fibration of $\partial Y$ to an $S^{1}$-fibration without any new special Seifert fibers. In this way there is a canonical choice for the Euler number of the 'middle' vertex, which is the 'middle' Euler number of the plumbing graph of $\bar{Y}$. We will calculate it below. Using this Euler number, the graph also determines a parametrization (framing) of $\partial Y \simeq S^{1} \times S^{1}$.

3.2. Homotopical properties of $Y$. In order to understand better the structure of $Y$ we consider its fundamental domain (in coordinates $(s, t, z)$ ): 


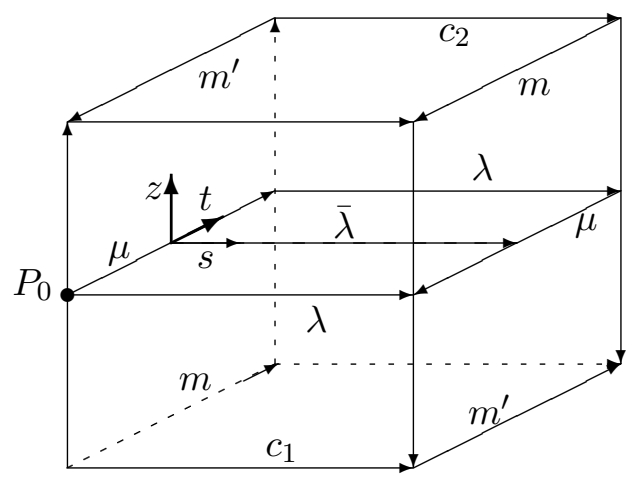

where $s \in[0, \pi], t \in[-\pi, \pi]$ and $z \in I=[-1,1]$. The original coordinates are $x=e^{s i}$ and $y=e^{t i}$. Then $Y$ is obtained by the following identification of the sides of the cube:

$$
(0, t, z) \sim(\pi,-t,-z) \text { and }(s,-\pi, z) \sim(s, \pi, z) .
$$

The boundary is

$$
\partial Y=\frac{[0, \pi] \times[-\pi, \pi] \times\{-1,1\}}{(0, t, \pm 1) \sim(\pi,-t, \mp 1),(s,-\pi, \pm 1) \sim(s, \pi, \pm 1)} \simeq S^{1} \times S^{1} .
$$

The $S^{1}$-action determining the canonical Seifert fibration (3.1.5) is induced by the translation along the $s$-axis. The exceptional fibers are

$$
\lambda=\{(s,-\pi, 0) \mid s \in[0, \pi]\}=\{(s, \pi, 0) \mid s \in[0, \pi]\} \text { and } \bar{\lambda}=\{(s, 0,0) \mid s \in[0, \pi]\} .
$$

Any fixed $(t, z) \notin\{(0,0),( \pm \pi, 0)\}$ determines a generic fibre in the form

$$
\{(s, t, z) \mid s \in[0, \pi]\} \cup\{(s,-t,-z) \mid s \in[0, \pi]\},
$$

where $(0, \pm t, \pm z)$ are glued together with $(\pi, \mp t, \mp z)$. For example, $c=c_{1} \cup c_{2} \subset \partial Y$ is a generic fibre.

The base space $D^{2}$ of the canonical Seifert-fibration can be represented as

$$
\frac{\{0\} \times[-\pi, \pi] \times[-1,0]}{(0,-\pi, z) \sim(0, \pi, z),(0, t, 0) \sim(0,-t, 0)} .
$$

Its boundary is the class of $m$, a circle.

Next, we describe the fundamental group and the homology of $Y$. $Y$ is homotopically equivalent with the Klein bottle $\mathcal{K}$. Let us choose the base point $P_{0}=(0,-\pi, 0)$. Both four vertexes of the rectangle representing $\mathcal{K}$ represents $P_{0}$. Thus the fundamental group of $Y$ can be presented as

$$
\pi_{1}(Y)=\langle\mu, \lambda \mid \mu \cdot \lambda \cdot \mu=\lambda\rangle,
$$

where $\mu$ and $\lambda$ denote also the class of $\mu$ and $\lambda$ in $\pi_{1}(Y)=\pi_{1}(\mathcal{K})$; cf. with the description (2) from 3.1). A more precise description can be given via the next diagrams, provided by the $\{z=0\}$ subspace of $Y$ (which can be identified with $\mathcal{K}$ ).

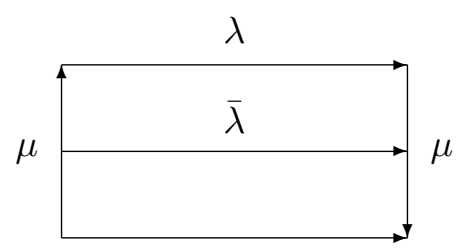

$\lambda$

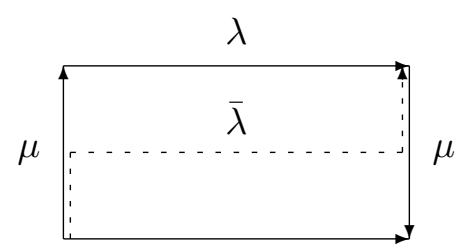

$\lambda$

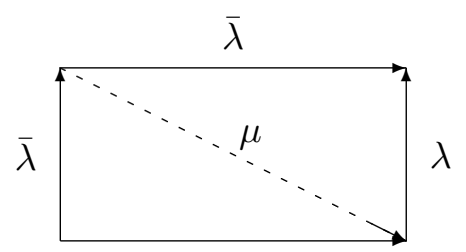

$\lambda$ 
The first diagram shows homological cycles. In order to rewrite the fundamental group, let $\bar{\lambda}$ be the closed path shown in the second diagram by the dashed line. Then $\bar{\lambda}=\mu \cdot \lambda$ in $\pi_{1}(Y)$. Note that $\lambda^{2}=\mu \cdot \lambda \cdot \mu \cdot \lambda=\lambda \cdot \mu \cdot \lambda \cdot \mu$, thus $\lambda^{2}=\bar{\lambda}^{2}=\mu^{-1} \cdot \lambda^{2} \cdot \mu$ and this element commutes with $\mu$. The fundamental group can be also presented as

$$
\pi_{1}(Y)=\left\langle\lambda, \bar{\lambda} \mid \bar{\lambda}^{2}=\lambda^{2}\right\rangle,
$$

according to the third picture above.

On the other hand, the fundamental group of the boundary is

$$
\pi_{1}(\partial Y)=H_{1}(\partial Y, \mathbb{Z}) \cong \mathbb{Z}\langle m\rangle \oplus \mathbb{Z}\langle c\rangle .
$$

The $\partial Y \simeq S^{1} \times S^{1} \hookrightarrow Y$ embedding (which is homotically the same as the orientation covering $S^{1} \times S^{1} \rightarrow \mathcal{K}$ ) induces a monomorphism $\pi_{1}(\partial Y) \rightarrow \pi_{1}(Y)$. It is determined by the images of the generators, which are

$$
m \mapsto \mu \text { and } c \mapsto \lambda^{2}=\bar{\lambda}^{2} .
$$

A direct computation shows that $\left[\pi_{1}(Y), \pi_{1}(Y)\right]=\mathbb{Z}\left\langle\mu^{2}\right\rangle$ and

$$
H_{1}(Y, \mathbb{Z}) \cong \mathbb{Z}\langle\lambda\rangle \oplus \mathbb{Z}_{2}\langle\mu\rangle .
$$

Note that $m=m^{\prime}$ in $H_{1}(\partial Y, \mathbb{Z})$, and analysing $\{s=0\} \subset Y$ one obtains that $m=-m^{\prime}$ in $H_{1}(Y, \mathbb{Z})$. Hence the class of $m$ in $H_{1}(Y, \mathbb{Z})$ has order 2 , it is exactly $\mu$.

The next Lemma shows that the classes $m$ and $c$ in $H_{1}(\partial Y, \mathbb{Z})$ have certain universal properties with respect to the inclusion $\partial Y \subset Y$.

Lemma 3.2.5. (a) $\pm m$ are the unique primitive elements of $H_{1}(\partial Y, \mathbb{Z})$ with the property that their doubles vanish in $H_{1}(Y, \mathbb{Z})$.

(b) $\pm c$ are the unique primitive elements of $H_{1}(\partial Y, \mathbb{Z})=\pi_{1}(\partial Y)$ whose images in $\pi_{1}(Y)$ are in the center of $\pi_{1}(Y)$.

Proof. (a) is clear. For (b) first note that any element of $\pi_{1}(Y)$ can be written in the from $\lambda^{k} \mu^{l}$ for some $k, l \in \mathbb{Z}$, and then using this one verifies that the center of $\pi_{1}(Y)$ is $\left\langle\lambda^{2}\right\rangle$.

3.3. Homological properties of $\bar{Y}$. The closed Seifert 3-manifold $\bar{Y}$ considered in 3.1(4) is constructed as follows. First we consider a new disc $D_{\text {new }}^{2}$ and the trivial fibration $D_{\text {new }}^{2} \times S^{1}$. Then we past $m$ with the boundary of $D_{\text {new }}^{2}$ and we extend the canonical Seifert-fibration of $Y$ above this disc (as base space) of the trivial fibration $D_{\text {new }}^{2} \times S^{1}$. This leads to the Seifert fibred closed manifold

$$
\bar{Y}=\frac{Y \cup\left(D_{\text {new }}^{2} \times S^{1}\right)}{\partial Y \sim \partial D_{\text {new }}^{2} \times S^{1}, m \sim \partial D_{\text {new }} \times *, c \sim * \times S^{1}} .
$$

$H_{1}(\bar{Y}, \mathbb{Z})$ can be determined by the Mayer-Wietoris sequence of the decomposition (3.3.1):

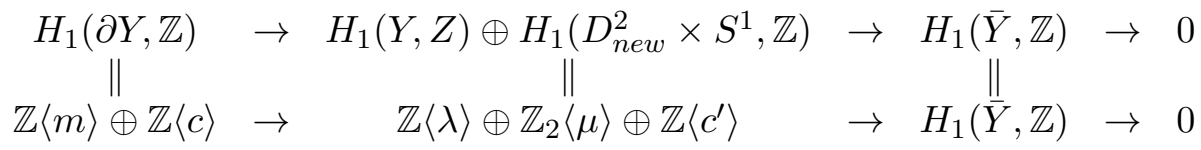

where $m \mapsto \mu$ and $c \mapsto 2 \lambda+c^{\prime}$. Thus

$$
H_{1}(\bar{Y}, \mathbb{Z}) \cong \mathbb{Z}\langle\lambda\rangle .
$$


3.4. A plumbing graphs of $Y$ and $\bar{Y}$. By [24], $\bar{Y}$ has a plumbing graph $G$ of the form

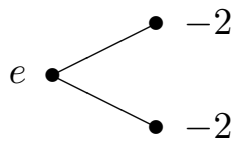

(see also the discussion from 3.1(4)) and the Euler number $e$ should be chosen such that $H_{1}\left(M^{3}(G), \mathbb{Z}\right) \cong \mathbb{Z}$ (cf. (3.3.2)). Here $M^{3}(G)$ denotes the plumbed 3-manifold associated with the graph $G$, it is the boundary of $M^{4}(G)$, the plumbed 4-manifold associated with $G$. This (via the long cohomological exact sequence of the pair $\left(M^{4}(G), M^{3}(G)\right)$ ) imposes the degeneracy of the intersection matrix of the plumbing (cf. e.g. $[22,15.1 .3]$ ). Hence $e=-1$. In particular, $\bar{Y}$ (without its Seifert fibration structure) is diffeomorphic to $S^{1} \times S^{2}$ (which also shows that $\bar{Y}$ admits an orientation reversing diffeomorphism).

Furthermore, consider the graph



The arrow denotes a knot $K \simeq S^{1} \subset \bar{Y}$, which is a generic $S^{1}$-fiber associated with the middle vertex by the plumbing construction of $\bar{Y}$. Let $N(K)^{\circ}$ be an open tubular neighborhood of $K$ in $\bar{Y}$. Then $Y \simeq \bar{Y} \backslash N(K)^{\circ}$, and the induced (singular/Seifert) $S^{1_{-}}$ fibration associated with the middle vertex (by the plumbing construction) on $Y$ agrees with the canonical Seifert fibration of $Y$. The Euler number -1 of the middle vertex determines a parametrization (framing) of $\partial Y \simeq S^{1} \times S^{1}$.

We can present $\pi_{1}(Y)$ also from the plumbing graph using the description of [19]. Let $\lambda$, $\bar{\lambda}$ and $c$ be oriented $S^{1}$-fibers associated with the three vertices provided by the plumbing construction (and extended by convenient connecting pathes to a base point as in [19]). Next, let $m$ be the meridian of $K$ corresponding to the arrowhead (and extended by a convenient path to the base point).

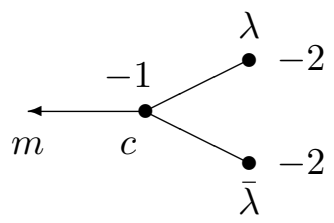

Then, by [19], there is a choice of the connecting pathes such that $\pi_{1}(Y)$ is generated by $\lambda, \bar{\lambda}, c$ and $m$, and they satisfies the relations $\lambda^{2}=\bar{\lambda}^{2}=c$, and $c=m \lambda \bar{\lambda}$. This is compatible with the description from subsection 3.2 , cf. (3.2.1) and (3.2.4).

Note that by plumbing calculus (cf. [23]) one has the equivalence of plumbed manifolds (where $\widetilde{\Gamma}$ is any graph):
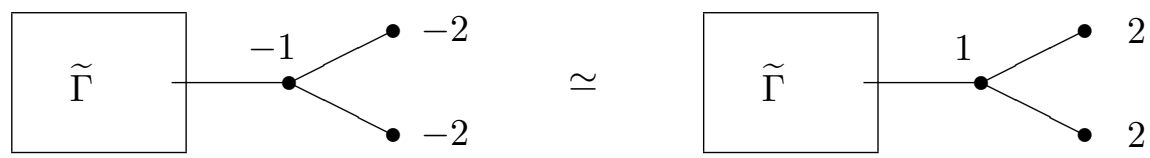

Hence, $Y$ or $-Y$ spliced along $K$ to any $3-$ manifold eventuate diffeomorphic manifolds.

\section{The boundary of the Milnor fibre}

4.1. Let $F=f^{-1}(\delta) \cap B_{\epsilon}^{6}$ be the Milnor fibre of $f$, where $\delta \in \mathbb{C}^{*},|\delta| \ll \epsilon$. We wish to construct the 3 -manifold $\partial F=f^{-1}(\delta) \cap S_{\epsilon}^{5}$ as a surgery of $S^{3}$ along the link $L$. 
Let $N_{i}$ be a sufficiently small tubular neighborhood of $L_{i}$ in $S^{3}$. For each $j=\{i, \sigma(i)\}$ we define $X_{j}$ as

$$
X_{j}=\left\{\begin{array}{cc}
S^{1} \times S^{1} \times I & \text { if } i \neq \sigma(i) \\
Y & \text { if } i=\sigma(i),
\end{array}\right.
$$

where $Y$ is the 3-manifold described in Section 3. Recall that $\partial Y \simeq S^{1} \times S^{1}$.

Proposition 4.1.2. One has an orientation preserving diffeomorphism

$$
\partial F \simeq\left(\mathfrak{S}^{3} \backslash \bigcup_{i=1}^{l} \operatorname{int}\left(N_{i}\right)\right) \cup_{\phi}\left(\bigcup_{j \in J} X_{j}\right),
$$

where $\phi: \partial\left(\mathfrak{S}^{3} \backslash \cup_{i} \operatorname{int}\left(N_{i}\right)\right) \rightarrow-\partial\left(\cup_{j \in J} X_{j}\right)$ is a collection $\left(\phi_{j}\right)_{j \in J}$ of diffeomorphisms

$$
\phi_{\{i, \sigma(i)\}}:\left\{\begin{array}{cl}
-\partial N_{i} \cup-\partial N_{\sigma(i)} \rightarrow-\partial\left(S^{1} \times S^{1} \times I\right) & \text { if } \quad i \neq \sigma(i) \\
-\partial N_{i} \rightarrow-\partial Y & \text { if } i=\sigma(i) .
\end{array}\right.
$$

Proof. The decomposition follows from the general decomposition proved in [25], see also $[22,2.3]$. For the convenience of the reader we sketch the construction. Recall that $\Upsilon_{j} \subset S_{\epsilon}^{5}$ is the link of the component $\Sigma_{j}$ of $\Sigma, j=\{i, \sigma(i)\} \in J$. Consider a sufficiently small tubular neighborhood $N\left(\Upsilon_{j}\right)$ of it in $S_{\epsilon}^{5}$. We can assume that $\Phi^{-1}\left(N\left(\Upsilon_{j}\right)\right)=N_{i} \cup N_{\sigma(i)}$. Furthermore, for $\epsilon$ small, the intersection of $\partial N\left(\Upsilon_{j}\right)$ with $K:=X \cap S_{\epsilon}^{5}$ is transversal. Therefore, for $0<|\delta| \ll \epsilon$, the intersection of $\partial N\left(\Upsilon_{j}\right)$ with $\partial F$ is still transversal in $S_{\epsilon}^{5}$, and, in fact, $K \backslash \cup_{j} N\left(\Upsilon_{j}\right)$ is diffeomorphic with $\partial F \backslash \cup_{j} N\left(\Upsilon_{j}\right)$. But, the former space can be identified via $\Phi$ by $\mathfrak{S}^{3} \backslash \cup_{i}\left(N_{i} \cup N_{\sigma(i)}\right)$. This is the space in the first parenthesis of (4.1.3).

The second one is a union of spaces of type $X_{j}:=\partial F \cap N\left(\Upsilon_{j}\right)$, which fibers over $\Upsilon_{j} \simeq S^{1}$. The fiber of the fibration is the Milnor fiber of the corresponding transversal plane curve singularity $\left(\right.$ of $\Sigma_{j}$ ). Since the transversal type is $A_{1}$, this fiber is $F_{j}:=S^{1} \times I$. The monodromy of the fibration is the so called geometric vertical monodromy of the transversal type, it is orientation preserving self-diffeomorphism of $S^{1} \times I$. If it does not permute the two components of $\partial F_{j}$ then it preserves the orientation of $I$, hence of $S^{1}$ too, hence up to isotopy it is the identity. If it permutes the components of $\partial F_{j}$ then up to isotopy it is $(\alpha, t) \rightarrow(\bar{\alpha},-t)$, where $(\alpha, t) \in S^{1} \times I$. The two types of vertical monodromies provide the two choices of $X_{j}$ in formula (4.1.1), cf. description (1) of $Y$ in subsection 3.1.

4.2. Preliminary discussion regarding the gluing. Our next aim is to describe the gluing functions $\phi_{j}$. In both cases two tori must be glued: if $i \neq \sigma(i)$ then basically one should identify $\partial N_{i}$ and $-\partial N_{\sigma(i)}$, otherwise $\partial Y$ and $\partial N_{i}$. Up to diffeotopy an orientation reversing diffeomorphism between tori is given by an invertible $2 \times 2$ matrix over $\mathbb{Z}$ with determinant -1 . It turns out that in our cases all these gluing matrices have the form

$$
\left(\begin{array}{cc}
-1 & n_{j} \\
0 & 1
\end{array}\right)
$$

hence its only relevant entry is the off-diagonal one.

This integer will be determined by a newly introduced invariant, the vertical index, associated with each $j \in J$. This is done using a special germ $H:\left(\mathbb{C}^{3}, 0\right) \rightarrow(\mathbb{C}, 0)$, which will have a double role. First, it provides some kind of framing along $\Sigma \backslash\{0\}$, and also helps to identify generators from the boundaries of $K \backslash \cup_{j} N\left(\Upsilon_{j}\right)$ and $\mathfrak{S}^{3} \backslash \cup_{i}\left(N_{i} \cup N_{\sigma(i)}\right)$ respectively (constructed in two different levels: in the target and in the source of $\Phi$ ).

We will use three parametrizations of $\partial N_{i} \approx S^{1} \times S^{1}$ with the same meridian but different longitudes. The topological longitude is the usual knot-theoretical Seifert-framing of $L_{i} \subset$ $S^{3}$. The resolution longitude is determined via a good embedded resolution of $(D, 0) \subset$ 
$\left(\mathbb{C}^{2}, 0\right)$, it creates the bridge with the decorations and the combinatorics of the resolution graph. Finally, the sectional longitude depends on $H$ and it will be used for describing the gluing. In fact, the sectional longitude allows us to compare the source and the target of $\Phi$ : being defined by the geometry of $H$, the function $H$ and its pull-back $\Phi^{*} H$ plays the role of transportation of the invariants from $\mathbb{C}^{3}$ level to $\mathbb{C}^{2}$ level.

4.3. The local form of $f$ along $\Sigma$. In the sequel we will use the notation $\Sigma^{*}=\Sigma \backslash\{0\}$ and $\Sigma_{j}^{*}=\Sigma_{j} \backslash\{0\}$. Recall that in a small neighborhood (in $\mathbb{C}^{3}$ ) of any point $p \in \Sigma^{*}$ the space $(X, 0)$ has two local components, both smooth and intersecting each other transversally.

A more precise local description along $\Sigma^{*}$ is the following. Let us fix a point $p_{0} \in \Sigma_{j}^{*}$ and let $U_{0}$ be a small neighborhood of $p_{0}$ in $\mathbb{C}^{3}$. In $U_{0}$ the function $f$ is a product $f_{1} \cdot f_{2}$, where both $f_{n}$ are holomorphic, $\left\{f_{n}=0\right\}$ are smooth and intersect each other transversally. (The intersection is $\Sigma^{*} \cap U_{0}$; later the fact that at $p_{0}$ the local parametrization of $\Sigma^{*} \cap U_{0}$ together with $f_{1}$ and $f_{2}$ might serve as local coordinates will be exploited further.)

$f_{1}$ and $f_{2}$ are well-defined up to a multiplication by an invertible holomorphic function $\iota$ of $U_{0}$; that is, $\left(f_{1}, f_{2}\right)$ can be replaced by $\left(\iota f_{1}, \iota^{-1} f_{2}\right)$. At any point $p \in \Sigma_{j}^{*} \cap U_{0}$ the linear term of $f_{n}, n \in\{1,2\}$, (say, in the Taylor expansion) is $T_{1}\left(f_{n}\right)=\sum_{k=1}^{3} u_{n k}(p)\left(x_{k}-p_{k}\right)$, where $\left(x_{1}, x_{2}, x_{3}\right)$ are the fixed coordinates of $\left(\mathbb{C}^{3}, 0\right)$. Let us code this in the non-zero vectors $u_{n}(p):=\left(u_{n 1}(p), u_{n 2}(p), u_{n 3}(p)\right)$. Hence, at any $p \in \Sigma_{j}^{*} \cap U_{0}$ we have two vectors $u_{1}(p)$ and $u_{2}(p)$ well-defined up to multiplication by $\left.\iota\right|_{\Sigma_{j}^{*} \cap U_{0}}$ (in the sense described above). Their classes $\left[u_{n}(p)\right] \in \mathbb{C P}^{2}$ for $p \in \Sigma_{j}^{*} \cap U_{0}$ are independent of the $\iota$-ambiguity, hence are well-defined elements. In particular, they determine a global pair of elements $\left[u_{1}(p)\right]$ and $\left[u_{2}(p)\right] \in \mathbb{C P}^{2}$ for $p \in \Sigma_{j}^{*}$, well-defined whenever $i \neq \sigma(i)$, and well-defined up to permutation whenever $i=\sigma(i)$.

In fact, we can do even more: there exists a splitting of $f$ into product $f_{1} \cdot f_{2}$ along $\Sigma_{j}^{*}$ without any invertible element ambiguity (but preserving the permutation ambiguity whenever $i=\sigma(i))$.

Indeed, assume that we are in the trivial covering $(i \neq \sigma(i))$ case, and let us cover $\Sigma_{j}^{*}$ by small discs $\left\{U_{\alpha}\right\}_{\alpha}$ such that on each $U_{\alpha}$ we can fix a splitting $f(p)=f_{1, \alpha}(p) \cdot f_{2, \alpha}(p)$, $p \in U_{\alpha}$. For any intersection $U_{\alpha \beta}=U_{\alpha} \cap U_{\beta}$ the two splittings can be compared: we define $\iota_{\alpha \beta} \in \mathcal{O}\left(U_{\alpha \beta}\right)^{*}$ by $\left.f_{1, \alpha}\right|_{U_{\alpha \beta}}=\left.\iota_{\alpha \beta} \cdot f_{1, \beta}\right|_{U_{\alpha \beta}}$. From this definition follows that $\left\{\iota_{\alpha \beta}\right\}_{\alpha, \beta}$ form a Čech 1-cocycle.

Lemma 4.3.1. $H^{1}\left(\Sigma_{j}^{*}, \mathcal{O}_{\Sigma_{j}^{*}}^{*}\right)=0$.

Proof. From the exponential exact sequence $0 \rightarrow \mathbb{Z} \rightarrow \mathcal{O} \rightarrow \mathcal{O}^{*} \rightarrow 0$ over $\Sigma_{j}^{*}$, we get that it is enough to prove the vanishing $H^{1}\left(\Sigma_{j}^{*}, \mathcal{O}_{\Sigma_{j}^{*}}\right)=0$, a fact which follows from Cartan's Theorem, since $\Sigma_{j}^{*}$ is Stein.

Since $H^{1}\left(\Sigma_{j}^{*}, \mathcal{O}_{\Sigma_{j}^{*}}^{*}\right)=0$, the cocycle $\left\{\iota_{\alpha \beta}\right\}_{\alpha, \beta}$ is a coboundary. This means that we can find invertible functions $\iota_{\alpha}$ on each $U_{\alpha}$ such that on $U_{\alpha \beta}$ one has $\left.\iota_{\alpha}\right|_{U_{\alpha \beta}}=\left.\iota_{\alpha \beta} \cdot \iota_{\beta}\right|_{U_{\alpha \beta}}$. This means that the local functions $\widetilde{f}_{1, \alpha}:=f_{1, \alpha} \cdot \iota_{\alpha}^{-1}, \widetilde{f}_{2, \alpha}:=f_{2, \alpha} \cdot \iota_{\alpha}$ on $U_{\alpha}$ provide a splitting (that is, $f(p)=\widetilde{f}_{1, \alpha}(p) \cdot \widetilde{f}_{2, \alpha}(p), p \in U_{\alpha}$ ), but in this new situation the local splittings glue globally: $\left.\widetilde{f}_{1, \alpha}\right|_{U_{\alpha \beta}}=\left.\widetilde{f}_{1, \beta}\right|_{U_{\alpha \beta}}$. If $i=\sigma(i)$ then we repeat the proof on $D_{i}$.

4.4. The special germ $H$. Next, we treat the 'aid'-germ $H$.

Definition 4.4.1. Let us fix $\Phi, f, \Sigma$ as above. A germ $H:\left(\mathbb{C}^{3}, 0\right) \rightarrow(\mathbb{C}, 0)$ is called transversal section along $\Sigma$ if $\Sigma \subset H^{-1}(0), H^{-1}(0)$ at any point of $\Sigma^{*}$ is smooth and intersects both local components of $(X, 0)$ transversally. 
We claim that transversal sections always exist.

Proposition 4.4.2. There exist complex numbers $a_{1}, a_{2}, a_{3}$ such that $H=a_{1} \partial_{x_{1}} f+a_{2} \partial_{x_{2}} f+$ $a_{3} \partial_{x_{3}} f$ is a transversal section.

Proof. For such $H$ one has $T_{1}(H)=\sum_{k=1}^{3} a_{k} u_{1 k} \cdot T_{1}\left(f_{2}\right)+\sum_{k=1}^{3} a_{k} u_{2 k} \cdot T_{1}\left(f_{1}\right)$. In particular, we have to show that for certain coefficients $\left\{a_{k}\right\}_{k}$ the expressions $\beta_{n}(p)=\sum_{k=1}^{3} a_{k} u_{n k}(p)$ (for $n \in\{1,2\}$ ) have no zeros for $p \in \Sigma^{*}$.

If $\gamma: X^{N} \rightarrow X$ is the Nash transform of $X$, then $\gamma^{-1}(0)$ is the set of limits of tangent spaces of $X \backslash\{0\}$, it is an algebraic set of $\mathbb{C P}^{2}$ of dimension $\leq 1$ (for details see e.g. [6] and references therein). The existence of Nash transform guarantees that $\left[\ell_{n}\right]=\lim _{p \rightarrow 0}\left[u_{n}(p)\right] \in$ $\mathbb{C P}^{2}$ exist. Indeed, the set $\left\{\left[l_{1}\right],\left[l_{2}\right]\right\}$ is the intersection of $\gamma^{-1}(0)$ with the strict transform of $\Sigma_{j}$. Then let $\left[a_{1}: a_{2}: a_{3}\right]$ be generic such that $\sum_{k} a_{k} \ell_{n k} \neq 0$ for $n \in\{1,2\}$. With this choice $\sum_{k=1}^{3} a_{k} u_{n k}(p) \neq 0$ for $p \neq 0$ and in a small representative of $\Sigma_{j}$.

Fix again $j=\{i, \sigma(i)\} \in J$, and let $p:(\mathbb{C}, 0) \rightarrow\left(\Sigma_{j}, 0\right) \subset\left(\mathbb{C}^{3}, 0\right), \tau \mapsto p(\tau)$, be a parametrization (normalization) of $\Sigma_{j}$. For any point $p_{0}=p\left(\tau_{0}\right)$ and neighborhood $\Sigma_{j}^{*} \cap U_{0} \ni p(\tau)$ the discussion from the second paragraph of 4.3 can be repeated, in particular we have the holomorphic vectors $u_{n}(p(\tau))(n \in\{1,2\})$ (with the choice ambiguities described there). Additionally, choose some $H$ as in Definition 4.4.1. The assumption regarding $H$ guarantees that $T_{1}(H)(p(\tau))=\beta_{1}(\tau) T_{1}\left(f_{1}\right)(p(\tau))+\beta_{2}(\tau) T_{1}\left(f_{2}\right)(p(\tau))$ for some holomorphic functions $\beta_{1}$ and $\beta_{2}$ on $p^{-1}\left(\Sigma_{j}^{*} \cap U_{0}\right)$. If we replace $\left(u_{1}, u_{2}\right)$ by $\left(\iota u_{1}, \iota^{-1} u_{2}\right)$ then $\left(\beta_{1}, \beta_{2}\right)$ will be replaced by $\left(\iota^{-1} \beta_{1}, \iota \beta_{2}\right)$, hence the product $\beta_{1} \beta_{2}$ is independent of all the $\iota$ and permutation ambiguities. It is a holomorphic function on $p^{-1}\left(\Sigma_{j}^{*} \cap U_{0}\right)$ depending only on the equations $f$ and $H$. This uniqueness also guarantees that taking different points of $\Sigma_{j}^{*}$ and repeating the construction, the output glues to a unique holomorphic function $\mathfrak{b}_{j}(\tau)$ on a small punctures disc of $(\mathbb{C}, 0)$. Usually $\mathfrak{b}_{j}(\tau)$ has no analytic extension to the origin, however one has the following.

Lemma 4.4.3. $\mathfrak{b}_{j}(\tau)$ is a Laurent series on $(\mathbb{C}, 0)$.

Proof. The finiteness of the pole follows e.g. from the homological identities from Theorem 4.5.4, or from Corollary 4.7.7 combined with (4.7.6).

We wish to emphasize that $\mathfrak{b}_{j}(\tau)$ and its pole order usually depends on the choice of $H$.

Definition 4.4.4. Let $a \tau^{\mathfrak{v}_{j}}$ (for some $a \in \mathbb{C}^{*}$ ) be the non-zero monomial with smallest power of $\tau$ in the Laurent series of $\mathfrak{b}_{j}(\tau)$. The integer $\mathfrak{v}_{j}$ is called the vertical index of $f$ along $\Sigma_{j}$ with respect to $H$ (or, the $H$-vertical index).

4.5. Computation of the gluing functions $\phi_{j}$. We fix a transversal section $H$ (cf. 4.4.1). Then the divisor $\Phi^{*}(H)$ is $H \circ \Phi=d \cdot d_{\sharp}$ for some (not necessarily reduced) germ $d_{\sharp}:\left(\mathbb{C}^{2}, 0\right) \rightarrow(\mathbb{C}, 0)$ (such that $d$ and $d_{\sharp}$ have no common components). Let $\left(D_{\sharp}, 0\right)$ be the (non-reduced) divisor associated with $d_{\sharp}$, and $N\left(L_{\sharp}\right)$ be a small tubular neighborhood of the reduced link $L_{\sharp}:=\operatorname{red}\left(D_{\sharp}\right) \cap \mathfrak{S}^{3}$ in $\mathfrak{S}^{3}$.

Let the Milnor fibre of $\Phi^{*}(H)$ (in $\mathfrak{S}^{3}$ ) be

$$
F_{\Phi^{*}(H)}=\left\{(s, t) \in \partial \mathfrak{B}_{\epsilon}=\mathfrak{S}^{3} \mid H(\Phi(s, t))>0\right\} .
$$

Let $\Lambda_{i}$ and $\Lambda_{\sharp}$ denote the components of the oriented intersection $F_{\Phi^{*}(H)} \cap \partial N_{i}$ and $F_{\Phi^{*}(H)} \cap$ $\partial N\left(L_{\sharp}\right)$ with the tubular neighborhood boundaries of $L_{i}$ and $L_{\sharp}$ respectively. ( $\Lambda_{\sharp}$ might have several components, in this notation we collect all of them.)

Furthermore, let $M_{i} \subset \partial N_{i}$ be an oriented meridian of $\partial N_{i}$ such that $\operatorname{lk}\left(M_{i}, L_{i}\right)=1$ and fix also (the oriented Seifert framing of $\left.D_{i}\right) L_{i}^{\prime} \subset \partial N_{i}$ with $\operatorname{lk}\left(L_{i}^{\prime}, L_{i}\right)=0$. (Here the linking numbers are considered in oriented $3-$ sphere $\mathfrak{S}^{3}$.) 
Definition 4.5.1. We call $L_{i}^{\prime}$ the topological longitude of the torus $\partial N_{i}$, while $\Lambda_{i}$ the sectional longitude of $\partial N_{i}$ associated with the transversal section $H$.

Clearly we have the following facts (where [·] denotes the corresponding homology class)

(a) $H_{1}\left(N_{i}, \mathbb{Z}\right) \cong \mathbb{Z}\left\langle\left[\Lambda_{i}\right]\right\rangle \cong \mathbb{Z}\left\langle\left[L_{i}^{\prime}\right]\right\rangle$

(b) $H_{1}\left(\partial N_{i}, \mathbb{Z}\right) \cong \mathbb{Z}\left\langle\left[\Lambda_{i}\right]\right\rangle \oplus \mathbb{Z}\left\langle\left[M_{i}\right]\right\rangle \cong \mathbb{Z}\left\langle\left[L_{i}^{\prime}\right]\right\rangle \oplus \mathbb{Z}\left\langle\left[M_{i}\right]\right\rangle$.

We want to express $\left[\Lambda_{i}\right]$ in terms of $\left[M_{i}\right]$ and $\left[L_{i}^{\prime}\right]$.

Lemma 4.5.3. Define $\lambda_{i}=-\Sigma_{k \neq i} D_{k} \cdot D_{i}-D_{\sharp} \cdot D_{i}$, where $C_{1} \cdot C_{2}$ denotes the intersection multiplicity of $\left(C_{1}, 0\right)$ and $\left(C_{2}, 0\right)$ at $0 \in \mathbb{C}^{2}$. Then $\left[\Lambda_{i}\right]=\left[L_{i}^{\prime}\right]+\lambda_{i} \cdot\left[M_{i}\right]$ in $H_{1}\left(\partial N_{i}, \mathbb{Z}\right)$.

Proof. First note that $\left[\Lambda_{i}\right]=\left[L_{i}^{\prime}\right]+\operatorname{lk}\left(\Lambda_{i}, L_{i}\right) \cdot\left[M_{i}\right]$. Write $F_{\Phi^{*}(H)}^{\prime}:=F_{\Phi^{*}(H)} \backslash\left(\operatorname{int}\left(\cup_{i} N_{i} \cup\right.\right.$ $\left.\left.N\left(L_{\sharp}\right)\right)\right)$. Then $0=\operatorname{lk}\left(\partial F_{\Phi^{*}(H)}^{\prime}, L_{i}\right)=\sum_{k} \operatorname{lk}\left(\Lambda_{k}, L_{i}\right)+\operatorname{lk}\left(\Lambda_{\sharp}, L_{i}\right)=\operatorname{lk}\left(\Lambda_{i}, L_{i}\right)+\sum_{k \neq i} D_{k}$. $D_{i}+D_{\sharp} \cdot D_{i}=\operatorname{lk}\left(\Lambda_{i}, L_{i}\right)-\lambda_{i}$.

Theorem 4.5.4. For any $j=\{i, \sigma(i)\} \in J$, the gluing functions $\phi_{j}$ from Proposition 4.1.2 is characterized up to homotopy by the following identities

Case 1: $i \neq \sigma(i)$. Identify the homology groups $H_{1}\left(S^{1} \times S^{1} \times\{1\}, \mathbb{Z}\right), H_{1}\left(S^{1} \times S^{1} \times\right.$ $\{-1\}, \mathbb{Z})$ and $H_{1}\left(S^{1} \times S^{1}, \mathbb{Z}\right)$ via the natural homotopies

$$
S^{1} \times S^{1} \times\{-1\} \stackrel{h}{\sim} S^{1} \times S^{1} \times[-1,1] \stackrel{h}{\sim} S^{1} \times S^{1} \times\{1\} .
$$

Then in this homology group one has

$$
\begin{gathered}
\phi_{j *}\left(\left[M_{i}\right]\right)=-\phi_{j *}\left(\left[M_{\sigma(i)}\right]\right) \quad \text { and } \\
\phi_{j *}\left(\left[\Lambda_{i}\right]\right)=\phi_{j *}\left(\left[\Lambda_{\sigma(i)}\right]+\mathfrak{v}_{j} \cdot \phi_{j *}\left[M_{\sigma(i)}\right]\right) .
\end{gathered}
$$

Case 2: $i=\sigma(i)$.

$$
\begin{aligned}
\phi_{j *}\left(\left[M_{i}\right]\right) & =-m \quad \text { and } \\
\phi_{j *}\left(\left[\Lambda_{i}\right]\right) & =c+\mathfrak{v}_{j} \cdot m,
\end{aligned}
$$

where $m$ and $c$ are the two generators of $H_{1}(\partial Y, \mathbb{Z})$, see 3.2 (especially (3.2.3) and (3.2.4)).

Proof. Recall that $\tau \mapsto p(\tau)$ is the normalization of $\Sigma_{j}$. At any point $p\left(\tau_{0}\right)$ of $\Sigma_{j}^{*}$ one can consider $\tau$ as a local complex coordinate, which can be completed with two other local complex coordinates $(x, y)$ (local coordinates in a transversal slice of $\Sigma_{j}$ at $p\left(\tau_{0}\right)$ ) such that $(\tau, x, y)$ form a local coordinate system of $\left(\mathbb{C}^{3}, p(\tau)\right)$, and locally $f(x, y)=x y$. These two local coordinates correspond to a splitting $f=f_{1} \cdot f_{2}$ of $f$. According to the discussion from 4.3 , the splitting of $f$ can be done globally along the whole $\Sigma_{j}^{*}$, hence these coordinated $(x, y)$ (corresponding to the components $f_{1}$ and $f_{2}$ ) can also be chosen globally along $\Sigma_{j}^{*}$ (with the permutation ambiguity whenever $i=\sigma(i)$, a fact which will be handled below).

Furthermore, in these coordinates, $T_{1} H=\beta_{1}(\tau) x+\beta_{2}(\tau) y$. Since $\beta_{1}(\tau)$ have no zeros and poles in the small representative of $\Sigma_{j}^{*},\left(\tau, \beta_{1} x, \beta_{1}^{-1} y\right)$ are also local coordinates, and in these coordinates the equations transform into $f(x, y)=x y$ and $T_{1} H(x, y)=x+\mathfrak{b}_{j}(\tau) y$.

If we concentrate on the points of $\Upsilon_{j}=\Sigma_{j} \cap S_{\epsilon}^{5}$, and its neighbourhood in $S_{\epsilon}^{5}$, then similarly as above, we have the real coordinate $\tau \in \Upsilon_{j}$, and the two complex (transversal) local coordinates $(x, y)$, with equations $f(x, y)=x y$ and $T_{1} H(x, y)=x+\mathfrak{b}_{j}(\tau) y$ as before.

Case 1. The above local description globalises as follows (compare also with the first part of the proof of Proposition 4.1.2). The space $\partial F \cap N\left(\Upsilon_{j}\right)$ has a product decomposition $\Upsilon_{j} \times F_{j}=S^{1} \times S^{1} \times I$, where $\Upsilon_{j}=S^{1}$ is the parameter space of $\tau$, and $F_{j}$ is the local Milnor fiber $F_{j}=\{x y=\delta\} \cap B_{\varepsilon}^{4}, 0<\delta \ll \varepsilon \ll \epsilon$, diffeomorphic to $S^{1} \times I$. In other words, $\partial F \cap N\left(\Upsilon_{j}\right)$ is the space $\left\{(\tau, x, y) \in S^{1} \times B_{\varepsilon}^{4}: x y=\delta\right\}, 0<\delta \ll \varepsilon \ll \epsilon$ (here we will use the same $\tau$ notation for the parameter of $S^{1}$ ). Note that for $\delta \ll \varepsilon$, the boundary of $F_{j}$ is 
'very closed' to the two circles $\{|x|=\varepsilon, y=0\}$ and $\{x=0,|y|=\varepsilon\}$ of $\partial B_{\varepsilon}^{4}$. Using isotopy in the neighborhoods of these two circles, $\partial F_{j}$ can be identified with these two circles (similarly as we identify via Ehresmann's fibration theorem the boundary of the Milnor fiber of an isolated singularity with the link), and in order to simplify the presentation, we will make this identification. Hence, the boundary components of $\partial F \cap N\left(\Upsilon_{j}\right)$ can be identifyed (by isotopy in $\partial N\left(\Upsilon_{j}\right)$ ) with $\partial_{i}:=\left\{(\tau, x, y): \tau \in S^{1},|x|=\varepsilon, y=0\right\}$ and $\partial_{\sigma(i)}:=\left\{(\tau, x, y): \tau \in S^{1}, x=0,|y|=\varepsilon\right\}$. (The choice of indices $i$ and $\sigma(i)$ is arbitrary and symmetric.) These two tori are identified homologically since they are boundary components of $S^{1} \times F_{j}$. In $\partial_{i}$ we have the meridian $\tilde{M}_{i}=\{(\tau, x, y): \tau=1,|x|=\varepsilon, y=0\}$, while in $\partial_{\sigma(i)}$ we have the meridian $\tilde{M}_{\sigma(i)}=\{(\tau, x, y): \tau=1, x=0,|y|=\varepsilon\}$ (both naturally oriented as the complex unit circle). Since $\partial\left(\{\tau=1\} \times F_{j}\right)=\tilde{M}_{i} \cup-\tilde{M}_{\sigma(i)}$, the first wished identity follows.

Now, we would like to study the intersection curve of $\partial_{i}$ and the Milnor-fibre of $H$ associated with a positive argument, that is $\tilde{\Lambda}_{i}:=\partial_{i} \cap\{H>0\}$. This curve is homotopic with $\partial_{i} \cap\left\{T_{1} H=x+\mathfrak{b}_{j}(\tau) y>0\right\}$ and also with $\partial_{i} \cap\left\{x+\tau^{\mathfrak{v}_{j}} y>0\right\}$ in $\partial_{i}$, cf. the definition 4.4.4. Similarly, $\tilde{\Lambda}_{\sigma(i)}:=\partial_{\sigma(i)} \cap\{H>0\}$ is homotopic with $\partial_{\sigma(i)} \cap\left\{x+\tau^{\mathfrak{v}_{j}} y>0\right\}$ in $\partial_{\sigma(i)}$.

Thus $\tilde{\Lambda}_{i}$ is homotopic with $\left\{(\tau, x, y): \tau \in S^{1},|x|=\varepsilon, y=0\right\} \cap\left\{x+\tau^{\mathfrak{v}_{j}} y>0\right\}=$ $\left\{(\tau, x, y): \tau \in S^{1}, x=\varepsilon, y=0\right\}$ and $\tilde{\Lambda}_{\sigma(i)}$ with $\left\{(\tau, x, y): \tau \in S^{1}, x=0, y=\varepsilon \tau^{-\mathfrak{v}_{j}}\right\}$. Hence homologically $\tilde{\Lambda}_{\sigma(i)}+\mathfrak{v}_{j} \tilde{M}_{\sigma(i)}$ is represented by the circle $\left\{(\tau, x, y): \tau \in S^{1}, x=\right.$ $0, y=\varepsilon\}$, which is homologous in $S^{1} \times F_{j}$ with $\tilde{\Lambda}_{i}$. This is the second identity. Obviously, these identities can be transferred from the boundary $\partial_{i} \cup \partial_{\sigma(i)}$ of $S^{1} \times F_{j}$ into similar identities in $\partial N_{i} \cup \partial N_{\sigma(i)}$, via the diagramm, where all the maps are orientation preserving diffeomorphisms (cf. the proof of 4.1.2):

$$
\begin{array}{cccc}
\Phi: \quad \mathfrak{S}^{3} \backslash \cup_{i} N_{i} & \rightarrow & K \backslash \cup_{j} N\left(\Upsilon_{j}\right) \\
\partial N_{i} \sqcup \partial N_{\sigma(i)} & \rightarrow & \partial_{i} \sqcup \partial_{\sigma(i)} \\
\Lambda_{i}, \Lambda_{\sigma(i)} & \rightarrow & \tilde{\Lambda}_{i}, \tilde{\Lambda}_{\sigma(i)} \\
M_{i}, M_{\sigma(i)} & \rightarrow & \tilde{M}_{i}, \tilde{M}_{\sigma(i)} .
\end{array}
$$

Case 2. We use similar notations and conventions as in Case 1. Let us parametrize $\Upsilon_{j}$ as $\tau=e^{2 i s}, s \in[0, \pi]$. Then $\partial F \cap N\left(\Upsilon_{j}\right)$ is $\left([0, \pi] \times F_{j}\right) / \sim$, where by $\sim$ we identify $(0, x, y) \sim(\pi, y, x)$ for all $(x, y) \in F_{j}$. Let us parametrize $F_{j}$ as $x=\sqrt{\delta} r e^{i t}$ and $y=$ $\sqrt{\delta} r^{-1} e^{-i t}$, where $t \in[-\pi, \pi]$ and $r \in\left[r_{0}, r_{1}\right]$ such that $r_{0} r_{1}=1$ and $\delta\left(r_{0}^{2}+r_{1}^{2}\right)=\varepsilon^{2}$. Denote $z=\log _{r_{1}} r$. Then we can parametrize $\partial F \cap N\left(\Upsilon_{j}\right)$ by $(s, t, z)$, thus $\partial F \cap N\left(\Upsilon_{j}\right)$ is just $([0, \pi] \times[-\pi, \pi] \times[-1,1]) / \sim$, where by $\sim$ we identify $(0, t, z) \sim(\pi,-t,-z)$ and $(s,-\pi, z) \sim$ $(s, \pi, z)$. We regard this as parametrization of $\partial F \cap N\left(\Upsilon_{j}\right)$ by $Y$, cf. 3.2.

Set

$$
\begin{gathered}
\tilde{M}_{j 1}=\left\{\left(\tau=1, x=\sqrt{\delta} r_{1} e^{i t}, y=\sqrt{\delta} r_{0} e^{-i t}\right)\right\} \text { and } \\
\tilde{M}_{j 2}=\left\{\left(\tau=1, x=\sqrt{\delta} r_{0} e^{-i t}, y=\sqrt{\delta} r_{1} e^{i t}\right)\right\} .
\end{gathered}
$$

The are the two oriented meridians of $\partial F \cap N\left(\Upsilon_{j}\right)$, parametrized by $t \in[-\pi, \pi]$. In terms of $(s, t, z)$ they are

$$
\begin{gathered}
\tilde{M}_{j 1}=\{(s=0, t, z=1)\}=\{(s=\pi,-t, z=-1)\} \subset \partial Y \text { and } \\
\tilde{M}_{j 2}=\{(s=0,-t, z=-1)\}=\{(s=\pi, t, z=1)\} \subset \partial Y,
\end{gathered}
$$

thus with the notations of $3.2, \tilde{M}_{j 1}=-m^{\prime}$ and $\tilde{M}_{j 2}=-m$.

Similarly as in Case 1 , by an isotopy in $\partial N\left(\Upsilon_{j}\right)$ the boundary $\partial_{j}$ of $\partial F \cap N\left(\Upsilon_{j}\right)$ can be identifyed with $\partial_{j 1} \cup \partial_{j 2}$, where

$$
\partial_{j 1}:=\{(s, x, y): s \in[0, \pi],|x|=\varepsilon, y=0\} \text { and }
$$




$$
\partial_{j 2}:=\{(s, x, y): s \in[0, \pi], x=0,|y|=\varepsilon\} .
$$

The two parts of $\partial_{j}$ are glued together along the image of the oriented meridians

$$
\begin{gathered}
\tilde{M}_{j 1}=\left\{\left(s=0, x=\varepsilon e^{i t}, y=0\right)\right\}=\left\{\left(s=\pi, x=0, y=\varepsilon e^{i t}\right)\right\} \text { and } \\
\tilde{M}_{j 2}=\left\{\left(s=0, x=0, y=\varepsilon e^{i t}\right)\right\}=\left\{\left(s=\pi, x=\varepsilon e^{i t}, y=0\right)\right\} .
\end{gathered}
$$

(See also the description in 3.2.) Since the oriented boundary of $\partial\left(\{\tau=1\} \times F_{j}\right)$ is $\tilde{M}_{j 1} \sqcup \tilde{M}_{j 2}$, $\tilde{M}_{j 1}$ is homologous with $-\tilde{M}_{j 2}$ in $F_{j} \subset Y$. On the other hand $\tilde{M}_{j 1}$ is homologous with $\tilde{M}_{j 2}$ in $\partial Y \subset Y$, thus $\left[\tilde{M}_{j 1}\right]=\left[\tilde{M}_{j 2}\right]$ is an order-2 element in $H_{1}(Y, \mathbb{Z})$.

Consider the closed curve $C$ obtained as union of $C_{1}$ and $C_{2}$, where

$$
\begin{gathered}
C_{1}=\{(s, x=\varepsilon, y=0): s \in[0, \pi]\}=\{(s, t=0, z=1): s \in[0, \pi]\}, \text { and } \\
C_{2}=\{(s, x=0, y=\varepsilon): s \in[0, \pi]\}=\{(s, t=0, z=-1): s \in[0, \pi]\} .
\end{gathered}
$$

Note that $C_{1}$ connects the points $A_{1}=(s=0, x=\varepsilon, y=0)=(s=0, t=0, z=1)$ with $B_{1}=(s=\pi, x=\varepsilon, y=0)=(s=\pi, t=0, z=1)$, while $C_{2}$ connects the points $A_{2}=(s=0, x=0, y=\varepsilon)=(s=0, t=0, z=-1)$ with $B_{2}=(s=\pi, x=0, y=\varepsilon)=$ $(s=\pi, t=0, z=-1)$. Since $A_{1} \sim B_{2}$ and $A_{2} \sim B_{1}$, they form a closed curve. Note that $[C]=[c]$ in $H_{1}(\partial Y, \mathbb{Z})$, see 3.2 .

Similarly as in Case 1, the function $H$ can be replaced by $x+\tau^{\mathfrak{v}_{j}} y$, hence its level set associated with a positive value determines the curve $\tilde{\Lambda}_{j}=\left\{x+\tau^{\mathfrak{v}_{j}} y>0\right\} \cap \partial_{j}$. This consists of two parts, $\tilde{\Lambda}_{j 1}$ and $\tilde{\Lambda}_{j 2}$, where

$$
\tilde{\Lambda}_{j 1}=\{(s, x=\varepsilon, y=0): s \in[0, \pi]\}=\{(s, t=0, z=1): s \in[0, \pi]\} \subset \partial_{j 1},
$$

while $\tilde{\Lambda}_{j 2}=\left\{\left(s, x=0, y=\varepsilon e^{-2 i s v_{j}}\right): s \in[0, \pi]\right\}$ equals

$$
\left\{\left(s, t=2 \mathfrak{v}_{j} s(\bmod [-\pi, \pi]), z=-1\right): s \in[0, \pi]\right\} \subset \partial_{j 2} .
$$

$\tilde{\Lambda}_{j n}$ has the same end-points as $C_{n}$, hence $\tilde{\Lambda}_{j 1}$ and $\tilde{\Lambda}_{j 2}$ form together a closed curve, as we expect. Furthermore, $\tilde{\Lambda}_{j}+\mathfrak{v}_{j} \tilde{M}_{j 2}$ is homologous in $\partial Y$ with $C$.

The source and the target are connected by the restriction of $\Phi$, which gives the orientation preserving diffeomorphisms:

$$
\begin{array}{cccc}
\Phi: \mathfrak{S}^{3} \backslash \cup_{i} N_{i} & \rightarrow & K \backslash \cup_{j} N\left(\Upsilon_{j}\right) \\
\partial N_{i} & \rightarrow & \partial_{j} \\
\Lambda_{i} & \rightarrow & \tilde{\Lambda}_{j} \\
M_{i} & \rightarrow & \tilde{M}_{j 1} \text { homologous with } \tilde{M}_{j 2}
\end{array}
$$

Since $C$ identifies with $c$ and $\tilde{M}_{j 1}$ and $\tilde{M}_{j 2}$ with $-m, \tilde{\Lambda}_{i}=c+\mathfrak{v}_{j} \cdot m$ follows.

4.6. The $H$-independent description of the gluing. The 'vertical index'.

Recall that the sectional longitudes $\Lambda_{i}$ and the corresponding $H$-vertical indexes $\mathfrak{v}_{j}$ depend on the choice of $H$. The goal of this paragraph is to replace $\left(M_{i}, \Lambda_{i}\right)$ by the $H-$ independent $\left(M_{i}, L_{i}^{\prime}\right)$ and $\mathfrak{v}_{j}$ by an $H$-independent number.

Definition 4.6.1. For any $j=\{i, \sigma(i)\}$ define $\mathfrak{v i}_{j}$ by

$$
\mathfrak{v i}_{j}:=\left\{\begin{array}{ccc}
\lambda_{i}+\lambda_{\sigma(i)}+\mathfrak{v}_{j} & \text { if } & i \neq \sigma(i) \\
\lambda_{i}+\mathfrak{v}_{j} & \text { if } & i=\sigma(i)
\end{array} .\right.
$$

We call $\mathfrak{v} \mathfrak{i}_{j}$ the vertical index of $\Sigma_{j}$.

The next statement follows from 4.5.3 and 4.5.4 (see also 4.2). 
Corollary 4.6.3. For $i \neq \sigma(i)$

and for $i=\sigma(i)$

$$
\begin{gathered}
\phi_{j *}\left(\left[M_{i}\right]\right)=-\phi_{j *}\left(\left[M_{\sigma(i)}\right]\right) \quad \text { and } \\
\phi_{j *}\left(\left[L_{i}^{\prime}\right]\right)=\phi_{j *}\left(\left[L_{\sigma_{i}}^{\prime}\right]\right)+\mathfrak{v i}_{j} \cdot\left[M_{\sigma(i)}\right]
\end{gathered}
$$

$$
\begin{aligned}
\phi_{j *}\left(\left[M_{i}\right]\right) & =-m \quad \text { and } \\
\phi_{j *}\left(\left[L_{i}^{\prime}\right]\right) & =c+\mathfrak{v i}_{j} \cdot m
\end{aligned}
$$

hold in the sense described in 4.5.4.

Corollary 4.6.4. The integer $\mathfrak{v i}_{j}$ does not depend on the choice of $H$, thus it is an invariant of $f$ and $\Sigma_{j}$.

4.7. The plumbing graph of $\partial F$. We construct a plumbing graph for $\partial F$ by modifying a good embedded resolution graph of $(D, 0) \subset\left(\mathbb{C}^{2}, 0\right)$. (For notations see subsection 2.3.) The gluing of the plumbing construction uses different set of longitudes (cf. [23]). First we define them and then we rewrite the above established identities regarding $\phi_{j *}$ in this language.

We choose small tubular neighborhoods $N\left(E_{v}\right)$ of $E_{v} \subset \widetilde{\mathbb{C}^{2}}$ such that

$$
\pi^{-1}\left(\mathfrak{S}^{3}\right) \simeq \partial\left(\bigcup_{v \in V} N\left(E_{v}\right)\right) \quad\left(\text { diffeomorphic with } \mathfrak{S}^{3} \text { via } \pi\right)
$$

after smoothing the corners of $\bigcup_{v \in V} N\left(E_{v}\right)$ ). The tubular neighborhood $N\left(E_{v}\right)$ of $E_{v} \subset \widetilde{\mathbb{C}^{2}}$ is a $D^{2}$ (real 2-disk) bundle over $E_{v}$ with Euler number $e_{v}$. We can choose this bundle structure in such a way that $\tilde{D}_{i}$ is one of the fibres of $N\left(E_{v(i)}\right)$ for each $i=1, \ldots, l$.

We choose another generic fibre $\mathcal{F}_{i} \simeq D^{2}$ of the bundle $N\left(E_{v(i)}\right)$ near $\tilde{D}_{i}$, and we set $L_{i}^{\pi}=\pi\left(\partial \mathcal{F}_{i}\right) \subset \mathfrak{S}^{3}$. By the choice of the bundle structure of $N\left(E_{v(i)}\right)$ and by the choice of the fibre $\mathcal{F}_{i}$ we can assume that $L_{i}^{\pi} \subset \partial N_{i}$.

Definition 4.7.1. $L_{i}^{\pi} \subset \partial N_{i} \subset \mathfrak{S}^{3}$ is called the resolution longitude of $L_{i} \subset \mathfrak{S}^{3}$ associated with the resolution $\pi$.

We fix a resolution longitude $L_{i}^{\pi}$ for each $L_{i}$. Clearly,

$$
H_{1}\left(N_{i}, \mathbb{Z}\right)=\mathbb{Z}\left\langle\left[L_{i}^{\pi}\right]\right\rangle \text { and } H_{1}\left(\partial N_{i}, \mathbb{Z}\right)=\mathbb{Z}\left\langle\left[L_{i}^{\pi}\right]\right\rangle \oplus \mathbb{Z}\left\langle\left[M_{i}\right]\right\rangle .
$$

The following facts are well-known (cf. [2]).

Proposition 4.7.3. (a) $l k\left(L_{i}^{\pi}, L_{i}\right)=m_{i}(v(i))$.

(b) $\left[L_{i}^{\pi}\right]=\left[L_{i}^{\prime}\right]+m_{i}(v(i)) \cdot\left[M_{i}\right]$ holds in $H_{1}\left(\partial N_{i}, \mathbb{Z}\right)$.

Corollary 4.7.4. $\left[L_{i}^{\pi}\right]=\left[\Lambda_{i}\right]+\left(m_{i}(v(i))-\lambda_{i}\right) \cdot\left[M_{i}\right]$ holds in $H_{1}\left(\partial N_{i}, \mathbb{Z}\right)$.

Definition 4.7.5. For any $j=\{i, \sigma(i)\}$ define $\alpha_{j}$ by:

$$
\alpha_{j}=\left\{\begin{array}{ccc}
-m_{i}(v(i))+\lambda_{i}-m_{\sigma(i)}(v(\sigma(i)))+\lambda_{\sigma(i)}+\mathfrak{v}_{j} & \text { if } & i \neq \sigma(i) \\
\lambda_{i}-m_{i}(v(i))+\mathfrak{v}_{j} & \text { if } & i=\sigma(i) .
\end{array}\right.
$$

Then Theorem 4.5.4 and Corollary 4.7.4 give the following.

Corollary 4.7.7. Case 1: For $i \neq \sigma(i)$ in $H_{1}\left(S^{1} \times S^{1}, \mathbb{Z}\right)$ the following identities hold:

$$
\phi_{j *}\left(\left[M_{i}\right]\right)=-\phi_{j *}\left(\left[M_{\sigma(i)}\right]\right) \quad \text { and } \quad \phi_{j *}\left(\left[L_{i}^{\pi}\right]\right)=\phi_{j *}\left(\left[L_{\sigma(i)}^{\pi}\right]+\alpha_{j} \cdot\left[M_{\sigma(i)}\right]\right) .
$$

Case 2: For $i=\sigma(i)$ in $H_{1}(\partial Y, \mathbb{Z})$ the following identities hold:

$$
\phi_{j *}\left(\left[M_{i}\right]\right)=-m \text { and } \phi_{j *}\left(\left[L_{i}^{\pi}\right]\right)=c+\alpha_{j} \cdot m .
$$


4.8. The construction of the plumbing graph. From the embedded resolution graph $\Gamma$ of $(D, 0) \subset\left(\mathbb{C}^{2}, 0\right)$ associated with the resolution $\pi$ we construct a plumbing graph $\widehat{\Gamma}$.

Recall that $\Gamma$ has $l$ arrowhead vertices representing the strict transforms of $\left\{D_{i}\right\}_{i=1}^{l}$, and the $i$-th arrowhead is supported (via a unique edge) by the vertex $v(i) \in V$. We obtain the plumbing graph $\widehat{\Gamma}$ from $\Gamma$ as follows.

(1) Fix $j=\{i, \sigma(i)\}$ and assume that $i \neq \sigma(i)$. Then we identify the two arrowheads and we replace it by a single new vertex of $\widehat{\Gamma}$. We define the Euler number of the new vertex by $\alpha_{j}$. Both two edges (which in $\Gamma$ supported the arrowheads) will survive as edges of this new vertex (connecting it with $v(i)$ and $v(\sigma(i))$ respectively), however one of them will have a negative sign, the other one a positive sign. By plumbing calculus, cf. [23, Prop. 2.1. $\mathrm{R} 0(\mathrm{a})]$, the choice of the edge which has negative sign - denoted by $\ominus$ - is irrelevant. (The edges without any decorations, by convention, are edges with positive sign.)

(2) Fix $j=\{i, \sigma(i)\}$ such that $i=\sigma(i)$. Then the arrowhead associated with $i=\sigma(i)$ will be replaced by a new vertex and it will be decorated by Euler number $\alpha_{j}$. Furthermore, to this new vertex we attach the plumbing graph of $Y$ (cf. 3.4) as indicated below. The edge connecting the new vertex and the graph of $Y$ will have a negative sign $\ominus$.

(3) We do all these modification for all $j \in J$, otherwise we keep the shape and decorations of $\Gamma$.

More precisely, if the schematic picture of $\Gamma$ is the following,

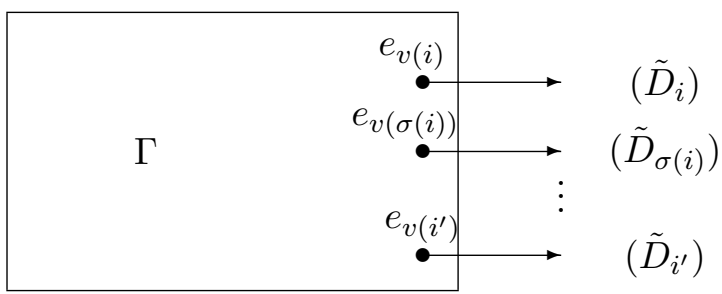

$$
\begin{aligned}
& (j=\{i, \sigma(i)\}, i \neq \sigma(i)) \\
& \left(j^{\prime}=\left\{i^{\prime}, \sigma\left(i^{\prime}\right)\right\}, i^{\prime}=\sigma\left(i^{\prime}\right)\right)
\end{aligned}
$$

then the schematic picture of $\widehat{\Gamma}$ is

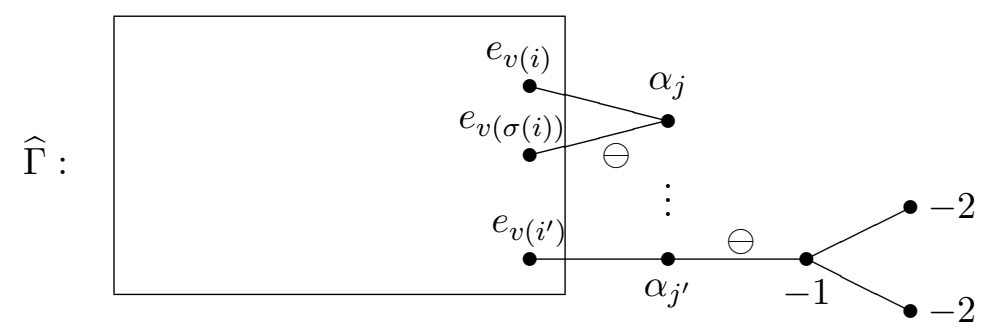

In fact, by plumbing calculus (cf. [23, Prop. 2.1. R0(a)]), whenever $i=\sigma(i)$ the edge sign from the newly created 'branch' (subtree) can be omitted, however at this point we put it since this is the graph provided by the proof (which reflects properly the corresponding base changes).

Note also that usually the graph $\widehat{\Gamma}$ (that is, the associated intersection form) is not negative definite (or, it is not even equivalent via plumbing calculus by a negative definite graph).

Above (when $i \neq \sigma(i)$ ) the coincidence $v(i)=v(\sigma(i))$ might happen, in fact, in all the cases we analyzed this coincidence (on minimal graph) does happen.

Theorem 4.8.1. The plumbing 3-manifold associated with the plumbing graph $\widehat{\Gamma}$ is orientation preserving diffeomorphic with $\partial F$. 
Proof. This follows from Corollary 4.7.7 and the relationships between this base-change and the plumbing construction as it is described in [23], pages 318-319. In the case $i=\sigma(i)$ the plumbing graph of $Y$ from 3.4 should be also used. Note that in both cases Corollary 4.7.7 provides a base change matrix from the left hand side of (4.8.2), this decomposes as a product as in the right hand side.

$$
\left(\begin{array}{cc}
-1 & \alpha_{j} \\
0 & 1
\end{array}\right)=\left(\begin{array}{ll}
0 & 1 \\
1 & 0
\end{array}\right)\left(\begin{array}{cc}
-1 & 0 \\
-\alpha_{j} & 1
\end{array}\right)\left(\begin{array}{cc}
0 & -1 \\
-1 & 0
\end{array}\right)
$$

This, according to [23, pages 318-320] is interpreted as a 'gluing' by a string (with length one), this is the new vertex (for each $j$ ) given by the constriction of $\widehat{\Gamma}$.

\section{The VERTICAL INDEX FOR $\Sigma^{1,0}$ TYPE GERMS}

5.1. $\Sigma^{1,0}$ type germs. Assume that $\Phi(s, t)=\left(s, t^{2}, t d(s, t)\right)$, where $d(s, t)=g\left(s, t^{2}\right)$ for some germ $g$, such that $d(s, t)$ is not divisible by $t$. In this case the equation of the image is $f=y g^{2}(x, y)-z^{2}=0$. These germs (more precisely, their $\mathcal{A}$-equivalence classes) are labeled by the Boardman symbol $\Sigma^{1,0}$, and they are exactly the corank-1 map germs with no triple points in their stabilization, see $[14,9]$ for details.

The set of double points is $D=\left\{(s, t) \in \mathbb{C}^{2} \mid d(s, t)=0\right\}$. It is equipped with the involution $\iota: D \rightarrow D, \iota(s, t)=(s,-t)$. The set of double values is

$$
\Sigma(f)=\left\{(x, y, z) \in \mathbb{C}^{3} \mid z=0 \text { and } g(x, y)=0\right\} .
$$

There are several options for the choice of the transversal section. E.g., Proposition 4.4.2 works with $a_{1}=a_{2}=a_{3}=1$. Furthermore, one can also take $H_{2}(x, y, z)=z$ or $H_{3}(x, y, z)=g(x, y)$.

If we take $H(x, y, z)=z$ then the $H$-vertical indexes $\mathfrak{v}_{j}$ are zero. This fact follows directly from the product decomposition of $f$. (However, for different other choices it can be nonzero as well.)

Proposition 5.1.1. For $\Sigma^{1,0}$-type germs $\Sigma_{j} \mathfrak{v i}_{j}=-\Sigma_{i \neq k} D_{i} \cdot D_{k}-C(\Phi)$, where $C(\Phi)$ is the number of crosscaps of a stabilization of $\Phi$, cf. $[21,16]$.

Proof. With the choice of $H=z, \Phi^{*}(H)=t d(s, t)$ and $\mathfrak{v}_{j}=0$. If $i \neq \sigma(i)$, then

$$
\mathfrak{v i}_{j}=-\Sigma_{k \notin\{i, \sigma(i)\}}\left(D_{i}+D_{\sigma(i)}\right) \cdot D_{k}-\left(D_{i}+D_{\sigma(i)}\right) \cdot\{t=0\},
$$

while for $i=\sigma(i)$ (see 4.6) one has

$$
\mathfrak{v i}_{j}=-\Sigma_{k \neq i} D_{i} \cdot D_{k}-D_{i} \cdot\{t=0\} .
$$

Taking the sum for all $j$ we get the identity, once we verify

$$
\Sigma_{i=1}^{l} D_{i} \cdot\{t=0\}=D \cdot\{t=0\}=\operatorname{dim}_{\mathbb{C}} \frac{\mathcal{O}_{\left(\mathbb{C}^{2}, 0\right)}}{\langle t, d(s, t)\rangle} .
$$

Here, the last identity follows from the algebraic definition of $C(\Phi)$, as the codimension of the Jacobian ideal of $\Phi$ (cf. $[16,21,10]$ ). In our case this ideal is genereted by $t$ and $d(s, t)$.

\section{EXAMPLES}

In the next paragraphs we provide some concrete examples. The families are taken from D. Mond's list of simple germs [16, Table 1]. In the sequel we provide the resolution graph of $D$ and the plumbing graph of $\partial F$. The computations are left to the reader.

The last two examples are more special than the previous ones: they are not of type $\Sigma^{1,0}$. In the first family $\left(H_{k}\right.$ from the list [16, Table 1$\left.]\right)$ the calculation has some nontrivial steps, hence we provide more details. The last example in 6.8 is a corank 2 map germ from [8]. 
6.1. Whitney umbrella, or cross-cap. $\Phi(s, t)=\left(s, t^{2}, t s\right)$ and $f(x, y, z)=x^{2} y-z^{2}=0$. The graph of $D$ is on the left, while our algorithm provides the graph from the right for $\partial F$
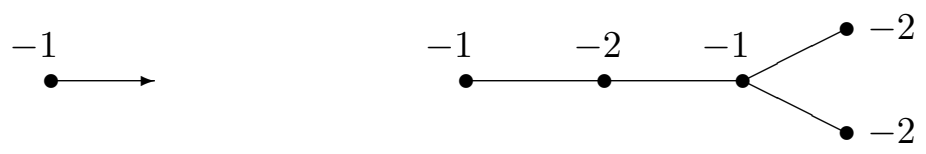

which after plumbing calculus transforms into $\stackrel{-4}{\bullet}$ (Compare also with Example 10.4.2 from [22].)

6.2. $S_{1}$. Set $\Phi(s, t)=\left(s, t^{2}, t^{3}+s^{2} t\right)$, hence $f(x, y, z)=y\left(y+x^{2}\right)^{2}-z^{2}$. The graph of $D$ is the first diagram, while the other two equivalent graphs represent $\partial F$
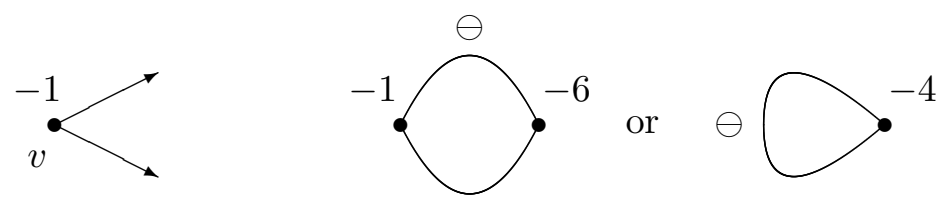

6.3. The family $S_{k-1}, k \geq 2$. One has $\Phi(s, t)=\left(s, t^{2}, t^{3}+s^{k} t\right)$ and $f(x, y, z)=y(y+$ $\left.x^{k}\right)^{2}-z^{2}=0$.

Case 1: $k=2 n$.

$D$ :

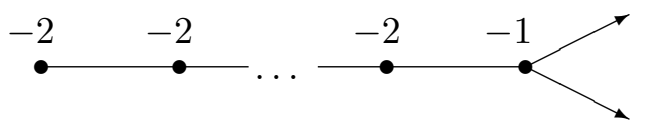

$\partial F$ :

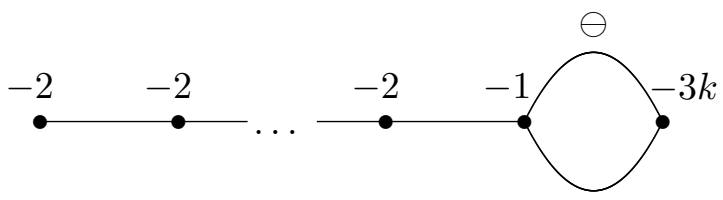

Here the number of $(-2)$-vertices is $n-1$.

Case 2: $k=2 n+1$.

$D$ :

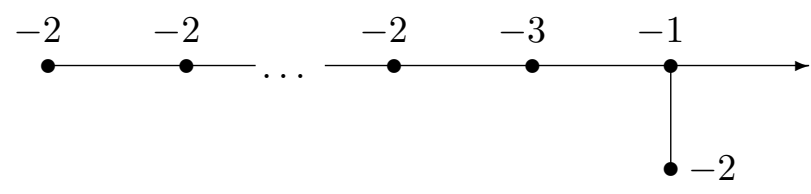

$\partial F$ :



where the number of $(-2)$-vertices on the left is $n-1$.

6.4. The family $B_{k}(k \geq 1) . \Phi(s, t)=\left(s, t^{2}, s^{2} t+t^{2 k+1}\right)$ and $f=y\left(x^{2}+y^{k}\right)^{2}-z^{2}=0$. The graph of $D$ is 
$D$ :

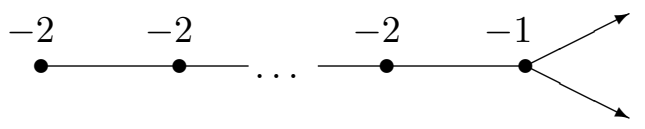

where the number of $(-2)$-vertices is $k-1$.

Note that the double point curve $D$ does not depend on the parity of $k$, however $\Sigma$ has one component, when $k$ is odd, and one component, when $k$ is even. Thus the pairing $\sigma$ changes the components of $D$ in the odd case, and $\sigma$ is the identity in the even case.

Case 1: $k=2 n+1$.

$\partial F$ :

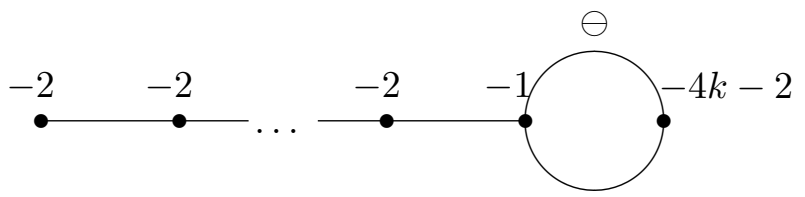

where the number of $(-2)$-vertices is $k-1$.

Case 2: $k=2 n$.

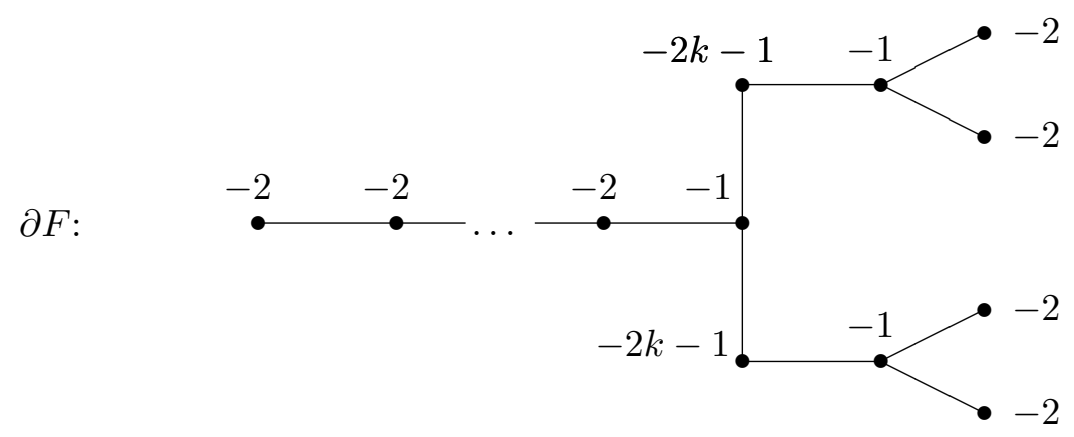

where the number of $(-2)$-vertexes on the left is again $k-1$.

6.5. The family $C_{k}(k \geq 1) . \Phi(s, t)=\left(s, t^{2}, s t^{3}+s^{k} t\right)$ and $f=y\left(x y+x^{k}\right)^{2}-z^{2}=0$.

Case 1: $k=2 n+1$.

$$
D \text { : }
$$
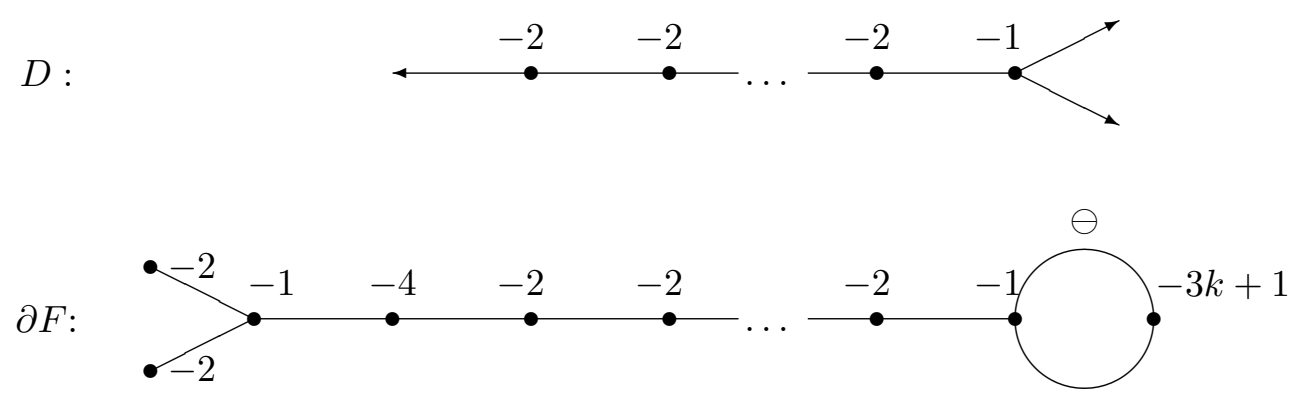

where the number of $(-2)$-vertices in the middle is $n-1$.

Case 2: $k=2 n$.

$$
D \text { : }
$$

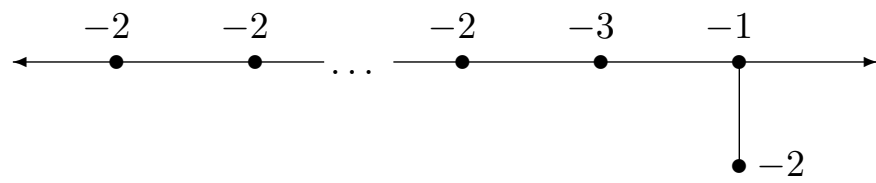


and the graph of $\partial F$ is

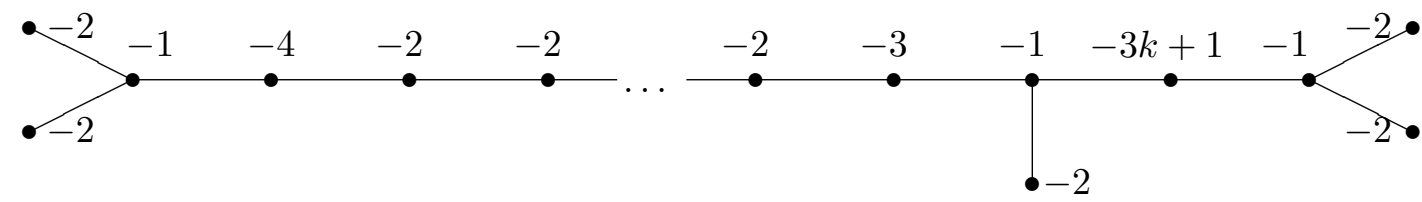

where the number of $(-2)$-vertices in the middle is $n-2$.

6.6. $F_{4}$. $\Phi(s, t)=\left(s, t^{2}, s^{3} t+t^{5}\right)$ and $f=y\left(x^{3}+y^{2}\right)^{2}-z^{2}=0$.

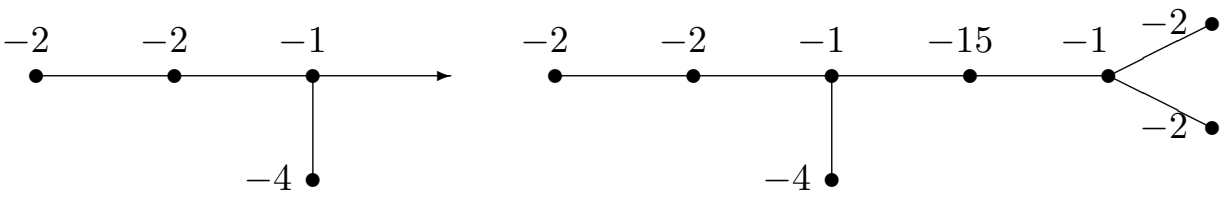

6.7. The family $H_{k}, k \geq 1$. In this case $\Phi(s, t)=\left(s, s t+t^{3 k-1}, t^{3}\right)$ and the equation of the image can be calculated as the $0^{t h}$ fitting ideal of $\Phi_{*}\left(\mathcal{O}_{\left(\mathbb{C}^{2}, 0\right)}\right)$. One obtains $f(x, y, z)=$ $z^{3 k-1}-y^{3}+x^{3} z+3 x y z^{k}=0$.

When $k>1$, the local form of $T^{2} f$ along $p(\tau)=\left(\tau^{3 k-2},-\tau^{3 k-1}, \tau^{3}\right) \in \Sigma^{*}$ is

$$
\begin{gathered}
\frac{\tau^{3 k-1}}{12 k^{2}-12 k+4}\left[-((3 k-3)-(3 k-1) \sqrt{3} i) \tau x^{\prime}+(3 k+(3 k-2) \sqrt{3} i) y^{\prime}+\left(6 k^{2}-6 k+2\right) \tau^{3 k-4} z^{\prime}\right] . \\
\cdot\left[-((3 k-3)+(3 k-1) \sqrt{3} i) \tau x^{\prime}+(3 k-(3 k-2) \sqrt{3} i) y^{\prime}+\left(6 k^{2}-6 k+2\right) \tau^{3 k-4} z^{\prime}\right],
\end{gathered}
$$

where $x^{\prime}=x-\tau^{3 k-2}, y^{\prime}=y+\tau^{3 k-1}, z^{\prime}=z-\tau^{3}$. With the choice $H(x, y, z)=\partial_{x} f(x, y, z)+$ $\partial_{y} f(x, y, z)+\partial_{z} f(x, y, z$, one has $\mathfrak{v}=3 k-1$.

For $k=1$ one gets

$$
T^{2}(f)=\left[z^{\prime}+\left(\frac{3}{2}+\frac{\sqrt{3}}{2} i\right) \tau y^{\prime}+\sqrt{3} i \tau^{2} x^{\prime}\right] \cdot\left[z^{\prime}+\left(\frac{3}{2}-\frac{\sqrt{3}}{2} i\right) \tau y^{\prime}-\sqrt{3} i \tau^{2} x^{\prime}\right],
$$

where $x^{\prime}=x-\tau, y^{\prime}=y+\tau^{2}, z^{\prime}=z-\tau^{3}$. In this case $\mathfrak{v}=0$.

In all cases $\lambda_{1}+\lambda_{2}+\mathfrak{v}=-3 k-1$.

$D:$

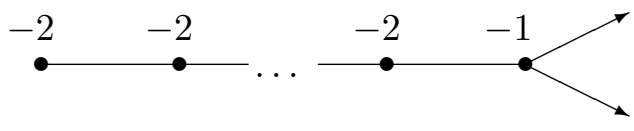

$\partial F$ :

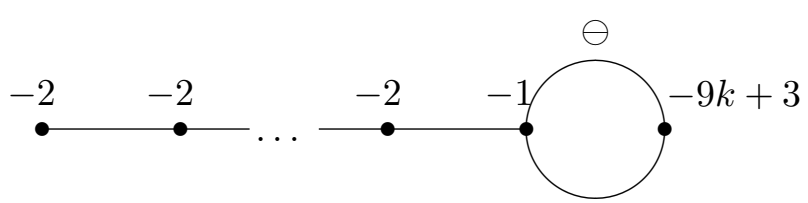

where the number of $(-2)$-vertexes is $3 k-3$.

In the special case $k=1$ the germs $H_{1}$ and $S_{1}$ analytic equivalent.

6.8. A corank 2 map germ. In this case $\Phi(s, t)=\left(s^{2}, t^{2}, s^{3}+t^{3}+s t\right)$ and $f(x, y, z)=$ $x^{6}-2 x^{4} y+x^{2} y^{2}-2 x^{3} y^{3}-2 x y^{4}+y^{6}-8 x^{2} y^{2} z-2 x^{3} z^{2}-2 x y z^{2}-2 y^{3} z^{2}+z^{4}=0$. 


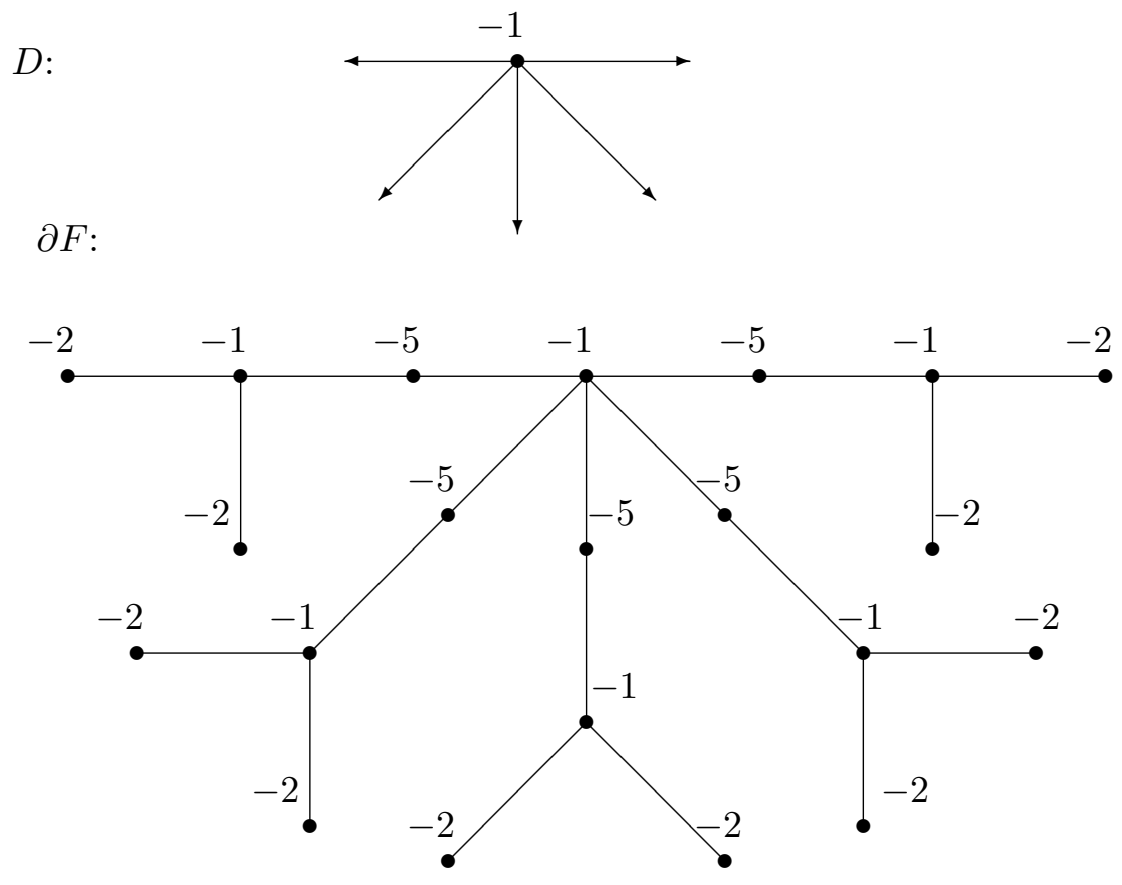

\section{REFERENCES}

[1] de Bobadilla, J. Fernández and Menegon Neto, A.: The boundary of the Milnor fibre of complex and real analytic non-isolated singularities, Geom. Dedicata 173 (2014), 143-162.

[2] Eisenbud, D. and Neumann, W.: Three-dimensional link theory and invariants of plane curve singularities, Annals of Math. Studies, 110, Princeton University Press, 1985.

[3] Durfee, A.H.: The signature of smoothings of complex surface singularities, Math. Ann. 232, no. 1 (1978), 85-98.

[4] Laufer, H.B.: Normal two-dimensional singularities. Annals of Math. Studies, 71, Princeton University Press, 1971.

[5] Laufer, H.B.: On $\mu$ for surface singularities, Proceedings of Symposia in Pure Math., 30 (1977), 45-49.

[6] Lê Dũng Tráng and Teissier, B.: Sur la geometrie des surfaces complexes I. Tangentes Exceptionnelles. Am. J. of Math. 101 (2) (1979), 420-452.

[7] Looijenga, E.: Isolated singular points on complete intersections, London Math. Society Lecture Note Series, volume 77 Cambridge Univ. Press, 1984.

[8] Marar, W. L. and Nuño-Ballesteros, J.J.: A note on finite determinacy for corank 2 map germs from surfaces to 3-space, Math. Proc. of the Cambridge Philosophical Society, 145, Issue 1 (2008), 153-163.

[9] Marar, W. L.; Nuño-Ballesteros, The doodle of a finitely determined map germ from $\mathbb{R}^{2}$ to $\mathbb{R}^{3}$, Advances in Mathematics, 221, (2009), 1281-1301.

[10] Marar, W. L.; Nuño-Ballesteros, J.J. and Peñafort-Sanchis, G.: Double point curves for corank 2 map germs from $\mathbb{C}^{2}$ to $\mathbb{C}^{3}$, Topology and its Applications, 159, (2012), 526-536.

[11] Michel, F. and Pichon, A.: On the boundary of the Milnor fiber of nonisolated singularities, Int. Math. Res. Not. 43 (2003), 2305-2311.

[12] Michel, F. and Pichon, A. and Weber, C.: The boundary of the Milnor fiber of Hirzebruch surface singularities, Singularity theory, World Scientific Publication (2007), 745-760.

[13] Milnor, J.: Singular points of complex hypersurfaces, Annals of Math. Studies, 61, Princeton University Press, 1968.

[14] Mond, D.: On the classification of germs of maps from $\mathbb{R}^{2}$ to $\mathbb{R}^{3}$, Proc. London Math. Soc. 50 (1985), $333-369$ 
[15] Mond, D.: Singularities of mappings from surfaces to 3-spaces, Singularity Theory, D.T. Lê, K. Saito, B. Teissier editors, World Scientific, 509-526.

[16] Mond, D.: Some remarks on the geometry and classification of germs of maps from surfaces to 3-space. Topology, 26(3) (1987), 361-383.

[17] Mond, D.: The number of vanishing cycles for a quasihomogeneous mapping from $\mathbf{C}^{2}$ to $\mathbf{C}^{3}$, Quart. J. Math. Oxford, 42 (1991), 335-345.

[18] Mond, D. and Pellikaan, R.: Fitting ideals and multiple points of analytic mappings, In Algebraic geometry and complex analysis, pages 107-161. Springer, 1989.

[19] Mumford, D.: The topology of normal singularities of an algebraic surface and a criterion for simplicity, Publ. Math. de l'IHES, 9(1) (1961), 5-22.

[20] Némethi, A.: Five lectures on normal surface singularities; lectures delivered at the Summer School in "Low dimensional topology", Budapest, Hungary 1998; Proc. of the Summer School, Bolyai Society Mathematical Studies 8 (1999), Low Dimensional Topology, 269-351.

[21] Némethi, A. and Pintér, G.: Immersions associated with holomorphic germs, Comment. Math. Helv. 90 (2015), 513-541.

[22] Némethi, A. and Szilárd, Á.: Milnor Fiber Boundary of a Non-isolated Surface Singularity, Lecture Notes in Math. 2037, Spriger 2012.

[23] Neumann, W. D.: A calculus for plumbing applied to the topology of complex surface singularities and degenerating complex curves, Trans. Am. Math. Soc. 268, 2 (1981), 299-344.

[24] Neumann, W. D. and Raymond, F.: Seifert manifolds, plumbing, $\mu$-invariant and orientation reversing maps, series Lecture Notes in Mathematics 664, (1978), 163-196.

[25] Siersma, D.: Variation mappings of singularities with 1-dimensional critical locus, Topology 30 (1991), 445-469.

[26] Sigurðsson, B.: The boundary of the Milnor fiber of the singularity $f(x, y)+z g(x, y)=0$, arXiv:1702.03752.

[27] Wahl, J.: Smoothings of normal surface singularities, Topology 20(3) (1981), 219-246.

[28] Wall, C. T. C.: Finite determinacy of smooth map-germs, Bull. London Math. Soc. 13 (1981), no. 6, 481-539.

Alfréd Rényi Institute of Mathematics, Hungarian Academy of Sciences, Reáltanoda utca 13-15, H-1053, Budapest, Hungary

ELTE - University of Budapest, Dept. of Geometry, Budapest, Hungary

BCAM - Basque Center for Applied Math., Mazarredo, 14 E48009 Bilbao, Basque Country SPAIN

E-mail address: nemethi.andras@renyi.mta.hu

A. Rényi Institute of Mathematics, 1053 Budapest, Reáltanoda u. 13-15, Hungary.

E-mail address: pinter.gergo@renyi.mta.hu 